\title{
The beneficial effects of different types of exercise interventions on motor and cognitive functions in older age: a systematic review
}

\author{
Oron Levin ${ }^{1}$, Yael Netz ${ }^{2}$ and Gal Ziv ${ }^{2^{*}}$
}

\begin{abstract}
The decline in cognitive and motor functions with age affects the performance of the aging healthy population in many daily life activities. Physical activity appears to mitigate this decline or even improve motor and cognitive abilities in older adults. The current systematic review will focus mainly on behavioral studies that look into the dual effects of different types of physical training (e.g., balance training, aerobic training, strength training, group sports, etc.) on cognitive and motor tasks in older adults with no known cognitive or motor disabilities or disease. Our search retrieved a total of 1095 likely relevant articles, of which 41 were considered for full-text reading and 19 were included in the review after the full-text reading. Overall, observations from the 19 included studies conclude that improvements on both motor and cognitive functions were found, mainly in interventions that adopt physical-cognitive training or combined exercise training. While this finding advocates the use of multimodal exercise training paradigms or interventions to improve cognitive-motor abilities in older adults, the sizeable inconsistency among training protocols and endpoint measures complicates the generalization of this finding.
\end{abstract}

Keywords: Exercise, Motor functions, Cognitive functions, Cognitive-motor training, Brain

\section{Background}

Changes in brain structure and function with age can give rise to a wide range of cognitive and motor declines in healthy older adults [1-13]; see reviews [12, 14-17]. Research over the past two decades has provided compelling evidence that these declines can be delayed or even reversed, and that skills can be revived by engaging in different sports activities and maintaining an active lifestyle; see reviews [18-22]. Given the relatively fast rise in the proportion of older adults in Europe and worldwide, finding new approaches or interventions to improve motor and cognitive functioning and promote healthy lifestyle is of importance. The present systematic review aims at providing a summary of research that has been conducted over the last decade and examined specifically the effect of different types of physical exercise training on both cognitive and motor functions.

\footnotetext{
* Correspondence: galziv@yahoo.com

${ }^{2}$ The Academic College at Wingate, Netanya, Israel

Full list of author information is available at the end of the article
}

In healthy older adults, regular physical exercise training has been reported to improve mood [23], relieve anxiety and depression [24], and enhance global cognitive functions such as memory [24-26], attention [24, 27], inhibition [27-33], and processing speed [22, 34]; see reviews $[21,22,35]$. Besides the beneficial impact of physical training on cognition, it has also been shown to improve mobility [29, 36-39], balance [37, 40], and fine upper limb control [41-44]; see reviews [39, 45, 46]. While there has been a growing number of studies evaluating the effects of physical exercise training on cognition in the past decade, the beneficial effects of training on motor functions per se have received less attention. In addition, the effects of physical exercise training on cognitive functions and motor functions have generally been explored separately. This segregation is somewhat surprising, given that motor and cognitive functions share similar brain network systems, and thus are expected to be influenced by parallel neurodegenerative processes in aging. 
For example, age-related changes in the structural and functional integrity of prefrontal and basal ganglia substructures have been reported to be associated with a range of cognitive deficits, such as a decline in memory [47], information processing speed $[6,9,11]$, and inhibition [4]; see [48, 49] of the involvement of the prefrontal-basal ganglia network in motor and cognitive functioning. Structural changes in the same substructures can also predict a wide range of motor declines, such as poor performance of complex coordination tasks $[3,8]$, longer action selection times [50], mobility deterioration [5], and balance loss [2]. Nonetheless, a growing body of evidence suggests that general physical training increases gray matter and white matter volume in prefrontal brain networks ([32, 51-55]; see reviews [20, 22]), which are compromised by aging processes to a greater extent than other regions of the brain $[10,56]$. However, note that prefrontal contributions to performance declines cannot be isolated from greater distributed gray and white matter loss in the whole brain [1, 57]. Taken together, these observations suggest that physical training could be an effective means to prevent brain atrophy and maintain (or even improve) cognitive and motor abilities in aging.

As physical activity appears to ameliorate cognitive decline in both healthy aging and age-related pathological conditions ([23, 24, 26, 27, 29-31, 37, 58, 59]; for review see [60]), questions emerge as to what extent improvements in cognitive functions predict gains in motor functions, and to what extent different types of exercise training differentially affect cognitive and motor functions. For example, it has been shown that exercise training reduced the need of prefrontal resources of executive function and attention involved in challenging treadmill walking. This, in turn, was speculated to allow older adults to allocate more attentional resources to processes related to balance control [27]. An alternative working hypothesis, nonetheless, would assume bilateral positive impacts of physical exercise training on both cognitive and motor functioning. Along these lines, the first aim of the current systematic review was to examine the specific beneficial effects of physical exercise interventions on cognitive and motor functioning in healthy older adult population. The second aim was to examine the interplay between cognitive and motor gains in relation to the physical exercise training used. In line with the aforementioned aims, our search strategy predominantly included search combinations of (i) common exercise interventions or training protocols such as cardiovascular (aerobic), strength and/or balance [18-46], [58, 59] and (ii) motor and cognitive tasks which are commonly used for evaluation of brainbehavior relationships in aging studies such as inhibition, reaction time, and balance control [1-17, 47-50]. We primarily focused on executive functions such as processing, attention, inhibition which have been shown crucial for successful performance of both gross and fine motor functioning such as locomotion, balance control, reaction time, and coordination; for review see [15-17].

\section{Literature search, selection process, data extraction, and quality assessment}

A systematic electronic search of the literature was carried out online through PubMed database that was published between January 2007 and December 2016. The search strategy was conducted by using a keyword search of the following terms:(physical activity OR training OR aerobic OR resistance OR strength OR dance OR yoga OR tai chi OR martial art OR qigong OR endurance OR balance OR cycling OR swimming OR running OR jogging OR walking OR cross country) AND ((cognitive OR cognition OR cognitive function* OR executive function* OR attention OR inhibition) AND (motor OR motor skill* OR motor task* OR motor learning OR reaction time (RT))) OR motor-cognitive. The search was conducted with the following additional filters: publication dates (10 years), age (65+ years), and pathology (NOT Parkinson's NOT stroke NOT Alzheimer NOT cancer NOT lesions* NOT patients NOT injury). A list of references, which included relevant reviews or original studies with no restrictions on study design and agerange, was also scanned for additional bibliography. Only studies published in English were considered.

The following inclusion criteria were implemented: (i) a longitudinal study design with at least two intervention groups (short-term or acute effect studies were not considered), (ii) physical intervention or combined physical and cognitive intervention (dual-task), and (iii) combined motor and cognitive outcomes as an endpoint. Studies were excluded if they: (i) were study design reports, (ii) did not include at least one cognitive function test and at least one motor function test at baseline or post-intervention phases of the study, (iii) were noninterventional or (iv) did not include at least one comparison group (i.e. single group pre- and post-test design) or cross-sectional study design. There were also restrictions with respect to the mean age ( $>65$ years old) and health condition of the included population (no reported neurodegenerative diseases, chronic illnesses and/or overt cognitive impairments).

A flow diagram of the study selection process is illustrated in Fig. 1. The search retrieved a total of 1095 likely relevant articles. All retrieved articles were screened by two reviewers (OL and GZ). Doubtful decisions for inclusion/exclusion were resolved by the senior co-author (YN). After screening by title and/or abstract, 1054 articles were excluded due to (i) topic irrelevance, (ii) being meta-analysis/review papers, (iii) irrelevant endpoint outcomes, (iv) the inclusion of one or more 


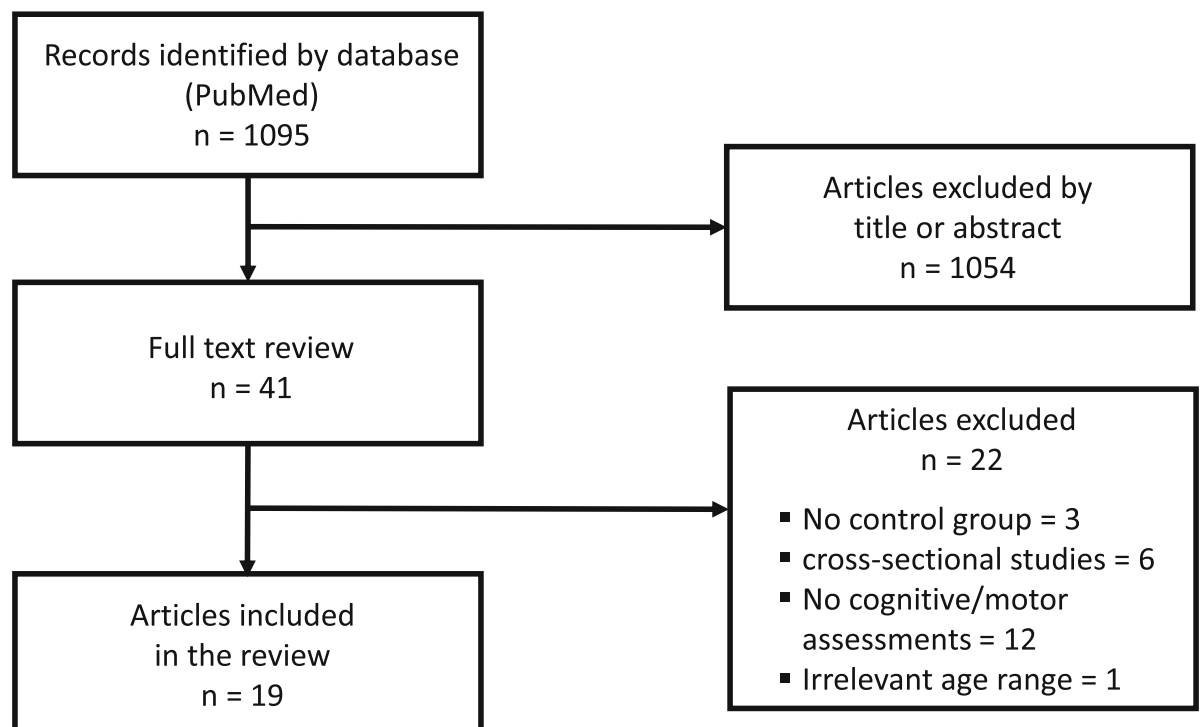

Fig. 1 Article selection process

patients groups, $(v)$ being a report of a study protocol with no actual collection of data, and (vi) absence of cognitive or motor assessments at baseline and/or endpoint. The remaining 41 articles were evaluated as potentially relevant papers and the full papers were obtained. After screening the full papers, 22 articles were excluded for the following reasons: irrelevant age-range [61], single group pre- and post-test design [62-64], a cross-sectional study design or no exercise intervention [65-70], or absence of cognitive or motor assessments at baseline or endpoint $[69,71-81]$. The remaining articles $(n=19)$ met all inclusion criteria and were included in the review [82-100]. Results from the aforementioned 19 articles were summarized with respect to: (i) demographic characteristics of participants (total sample size, number of group participants and gender ratio), (ii) characteristics of the intervention (exercise protocol, duration/frequency, and exercise intensity), and (iii) the outcome effects of the intervention on specific cognitive and motor functions. The aforementioned results are presented in Table 1. Lastly, article quality assessment was conducted using the Jadad scale [101] (see Table 2).

\section{Results}

\section{Sample characteristics}

The number of participants, mean age, and gender distribution for each intervention group in the 19 included studies are summarized in Table 1. Five studies had small sample sizes $(N<15)$ in one or more groups [83]: 2 groups, $N \leq 7$ per group; [85]: 2 groups, $N \leq 13$ per group; [86]: 2 groups, $N \leq 11$ per group; [87]: control group, $N=$ 13; [96]: physical-cognitive training group, $N=12$ ). In most of the included studies sample sizes per group were larger than 15, and in three studies sample sizes per group were equal to or larger than 50 [92, 97, 99]. In all studies the number of females was larger than that of males, however information about gender distribution within each intervention group was not always available. In one study [98], all of the included participants were female. Subject ages ranged from 55 to 97 years old and mean group ages ranged from $65.5 \pm 6.3$ [89] to $81.9 \pm 6.3$ years old [97].

\section{Interventions}

Studies included in this review reported multiple outcome measures, and an extensive range and diverse types of intervention protocols. The most frequent intervention protocol (11 of the 19 included studies) was combined exercise training (e.g. aerobic training followed by resistance training) [82-84, 88, 90, 91, 97-100]. The second most frequent intervention protocol (9 of 19 included studies) was combined physical-cognitive training. Here physical exercise training was either conducted simultaneously with a cognitive task in a dual-task manner [84, 91, 93, 94, 96, 100], or was followed by separate cognitive interventions [83, 92, 97]. The remaining intervention protocols consisted of single-exercise training paradigms, involving aerobic training $[89,95]$, resistance training $[85,89]$, balance training $[86,89]$ or dance $[87,88]$. Nine studies included a passive control group [85, 86, 89-91, 93, 94, 96, 98]. Alternatively, participants in control groups underwent health education classes $[87,92,99]$ or were subjected to lesser physical (or cognitive) training, for example training of gross motor activities [82] or training of a single cognitive task [96].

Types and durations of the interventions varied considerably between studies. The durations of the intervention period varied, ranging from 6 weeks $[85,86]$ to 


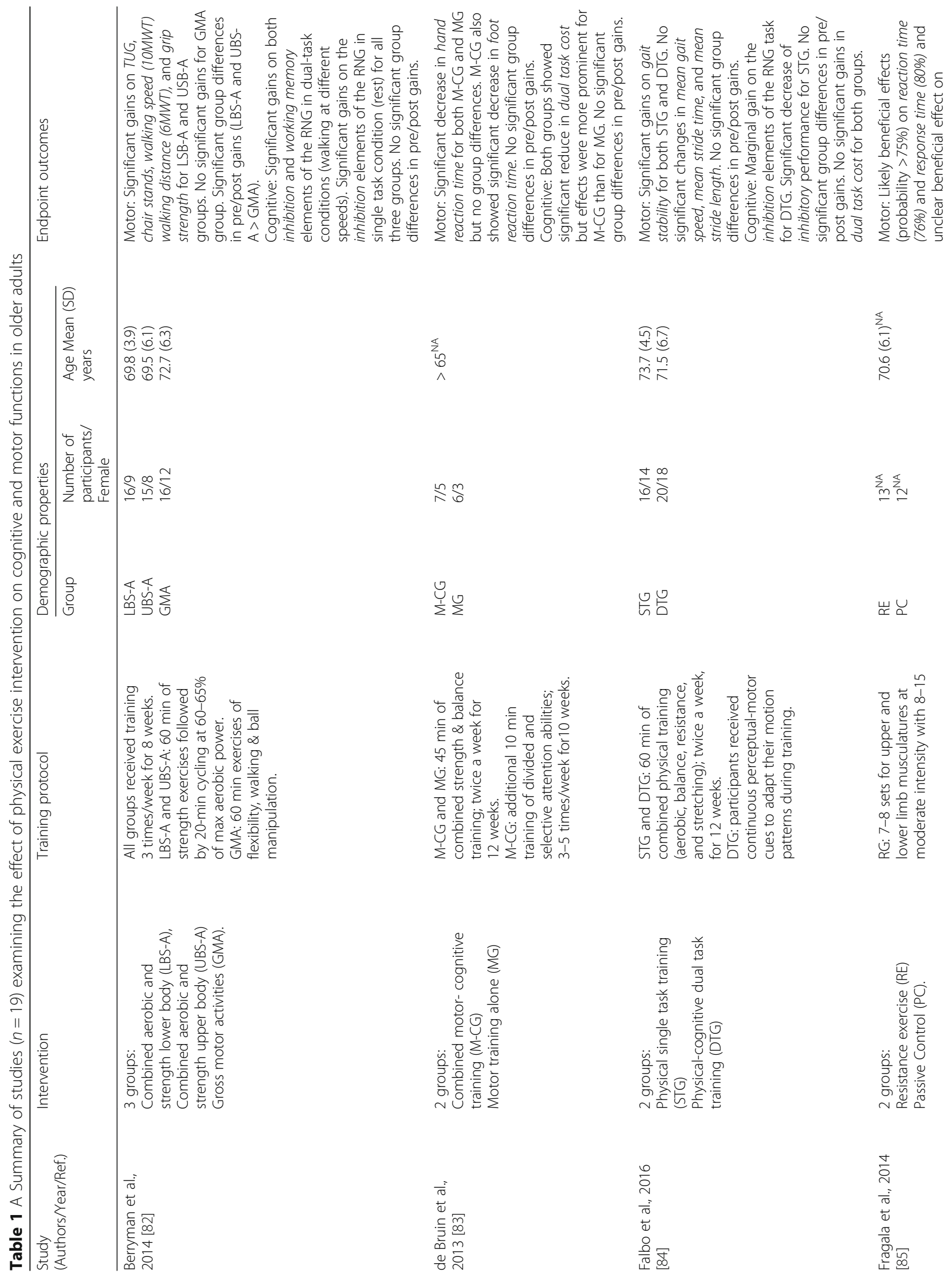




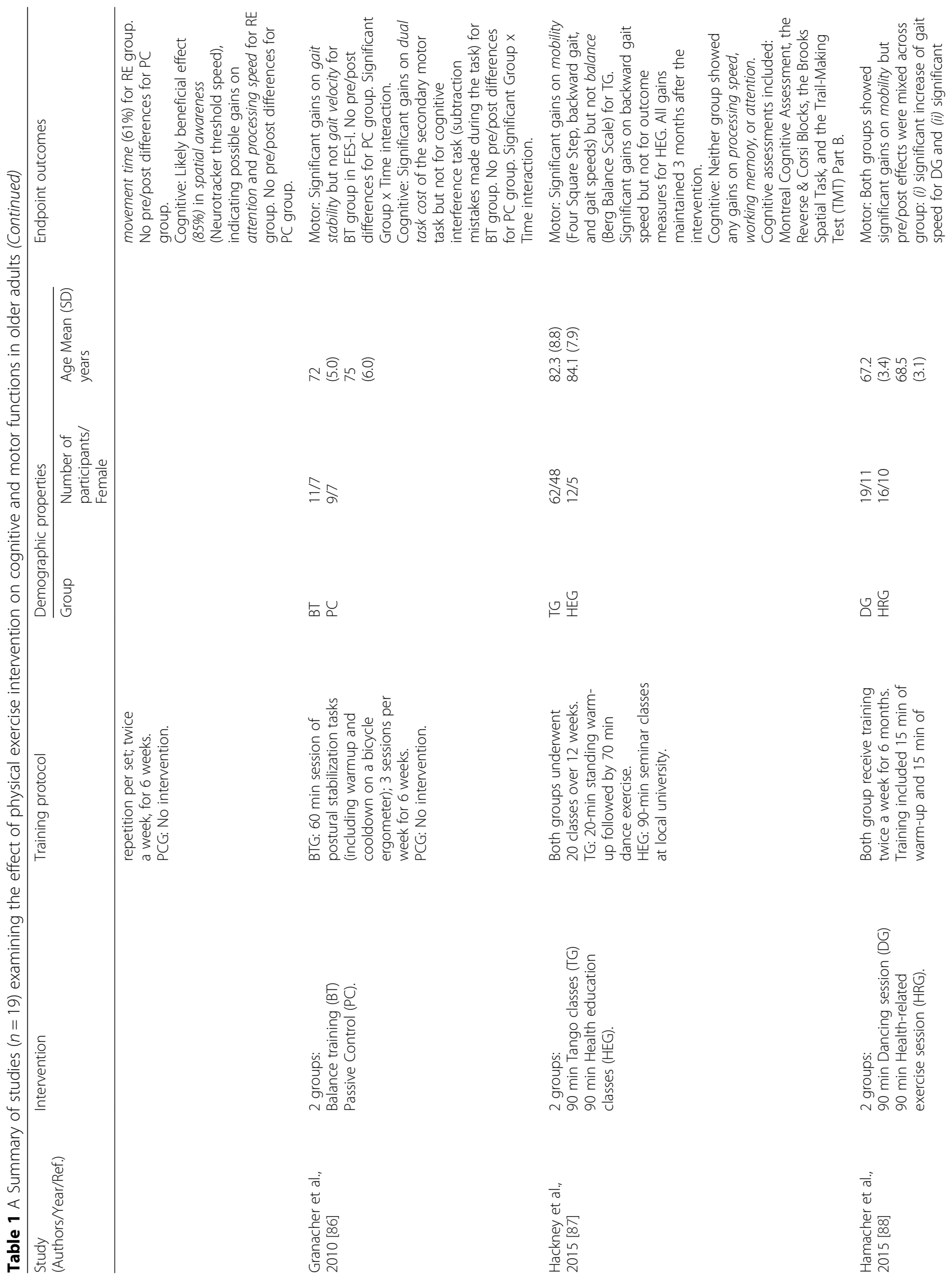




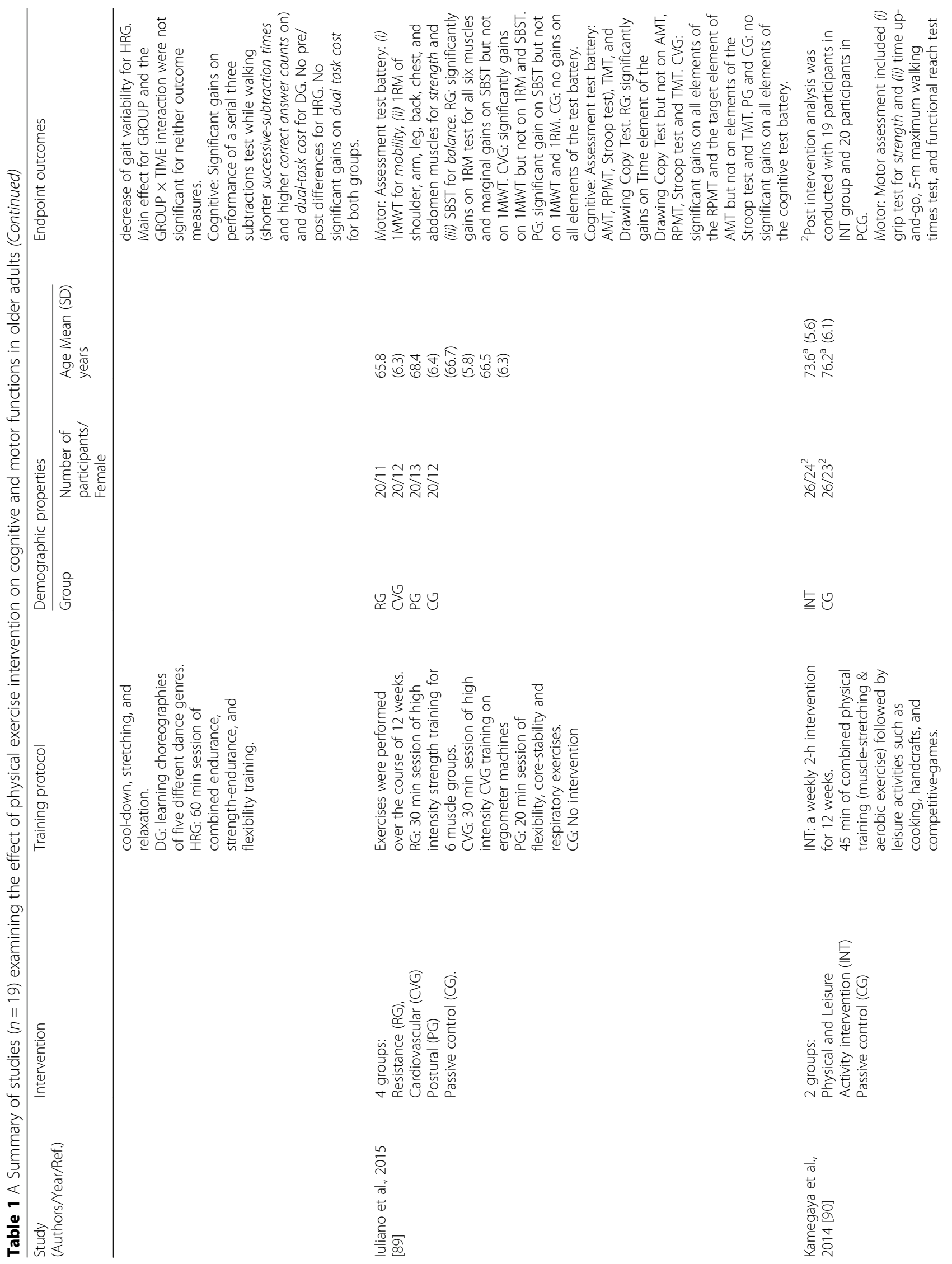




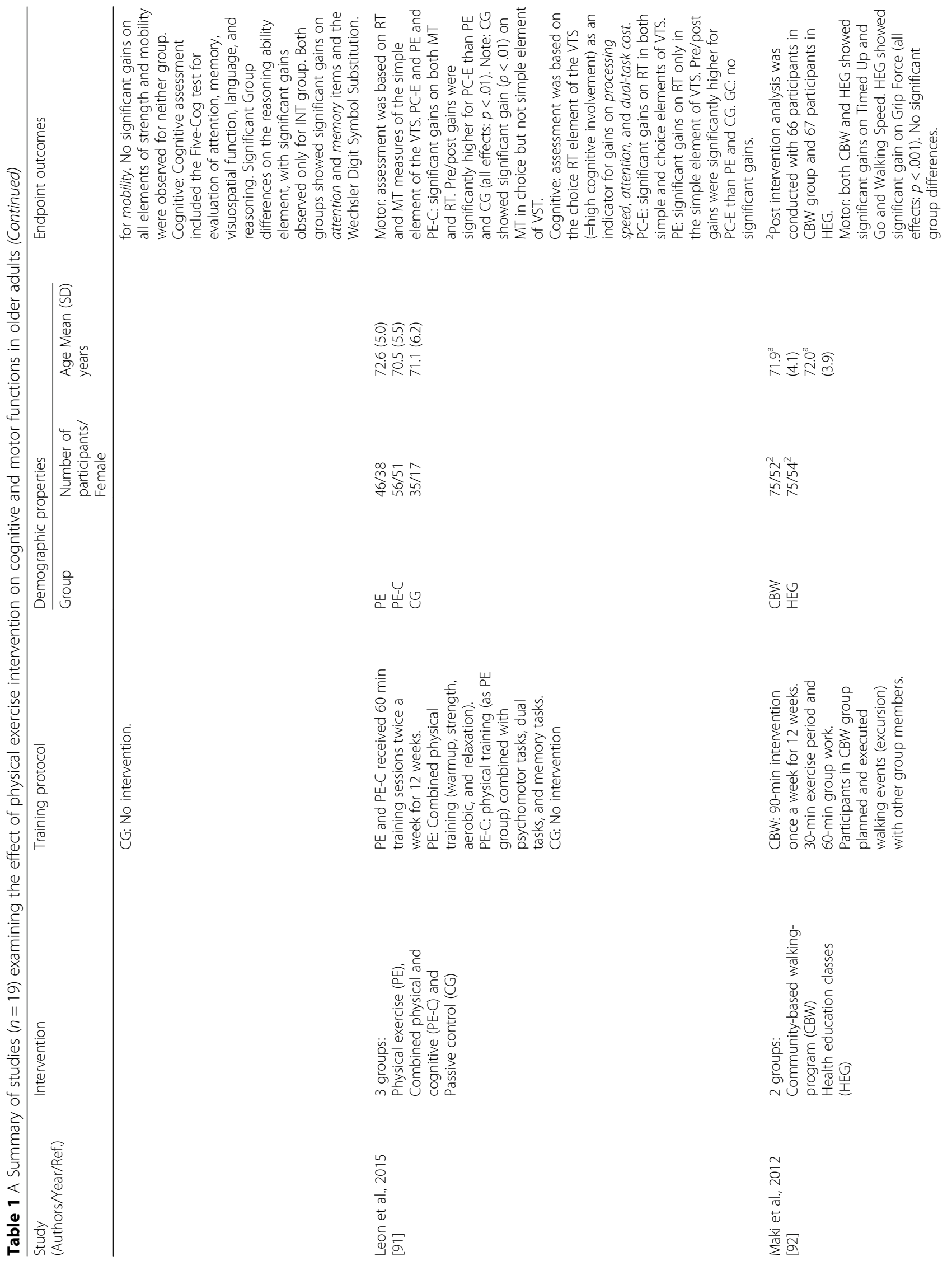




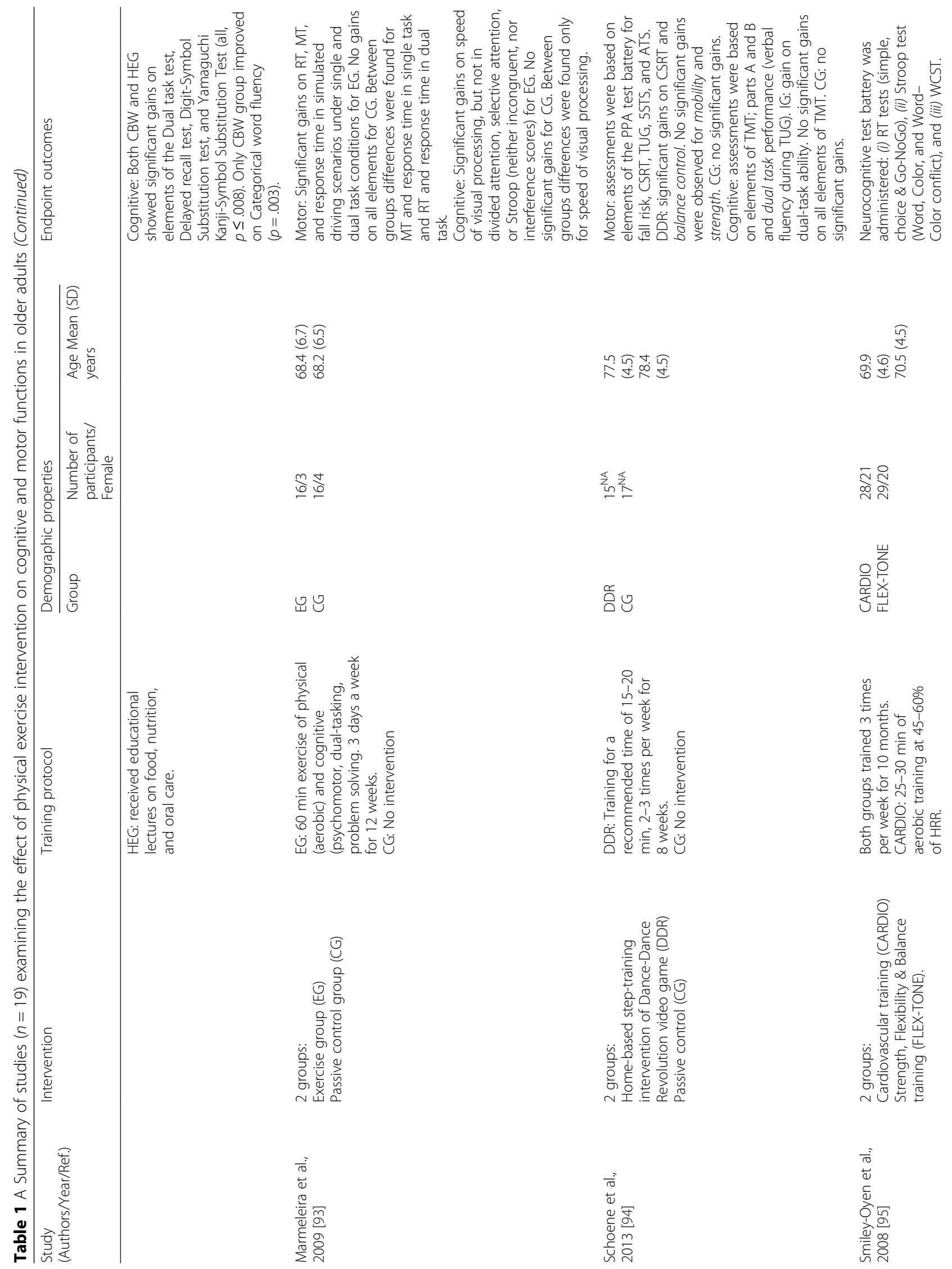




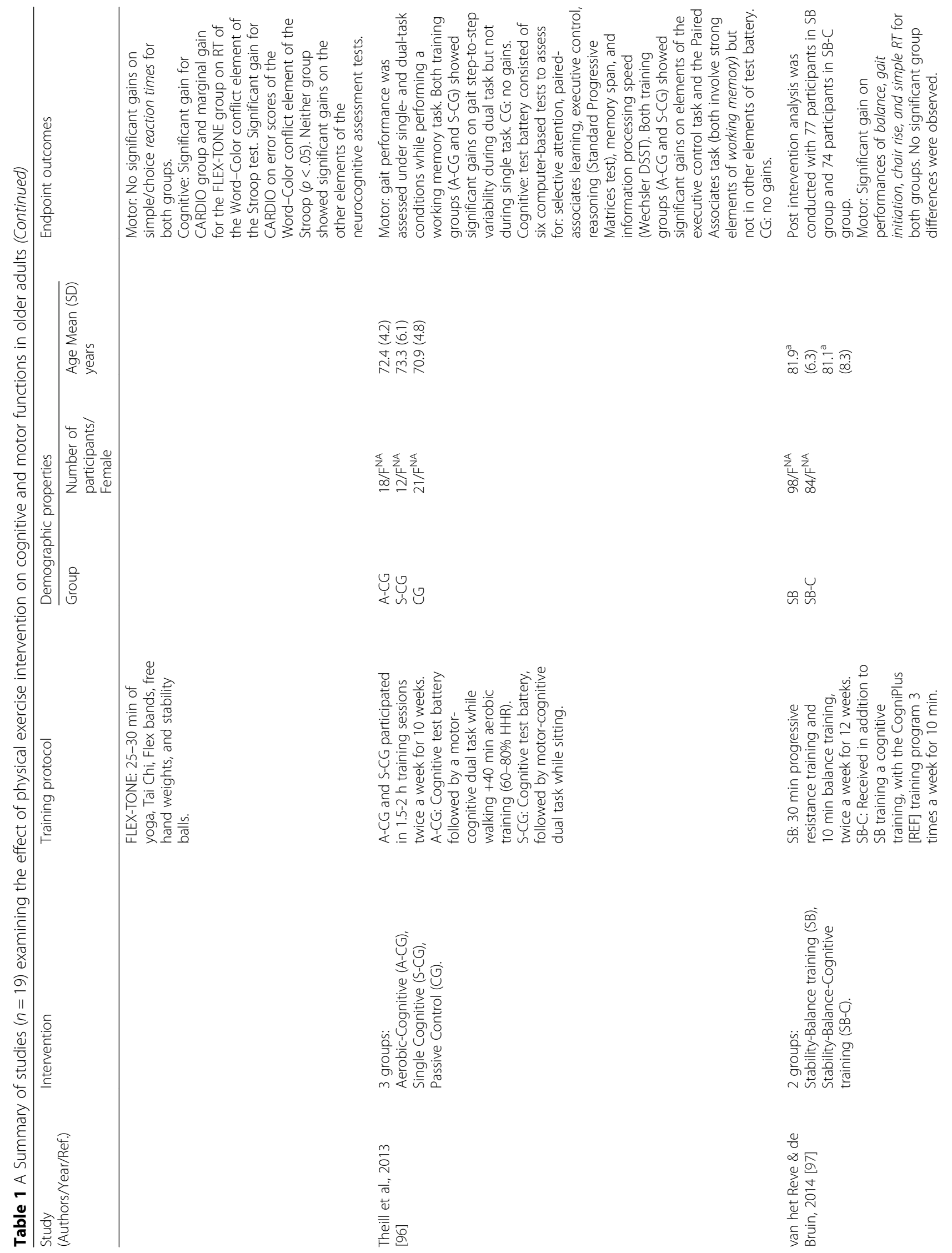




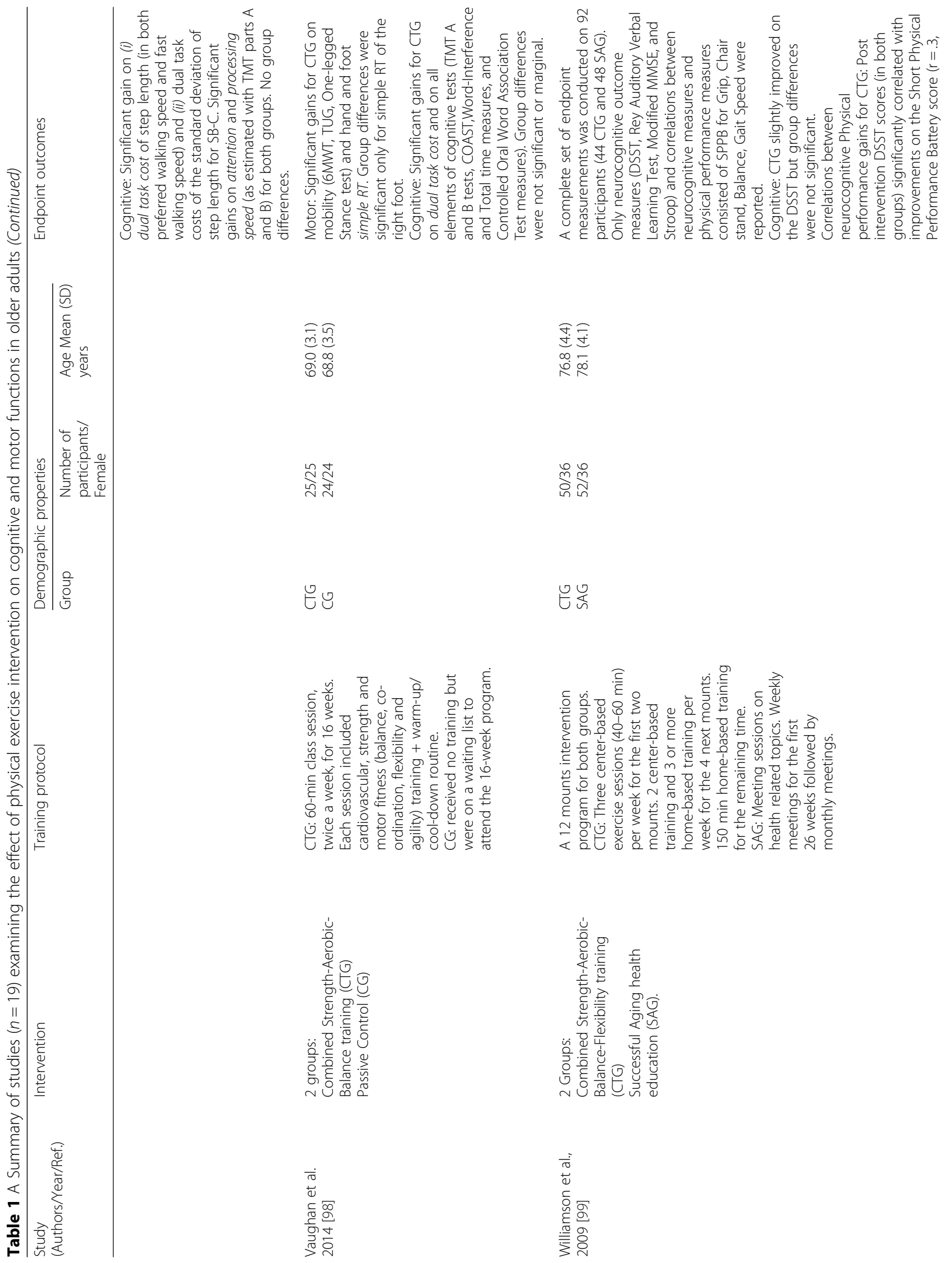




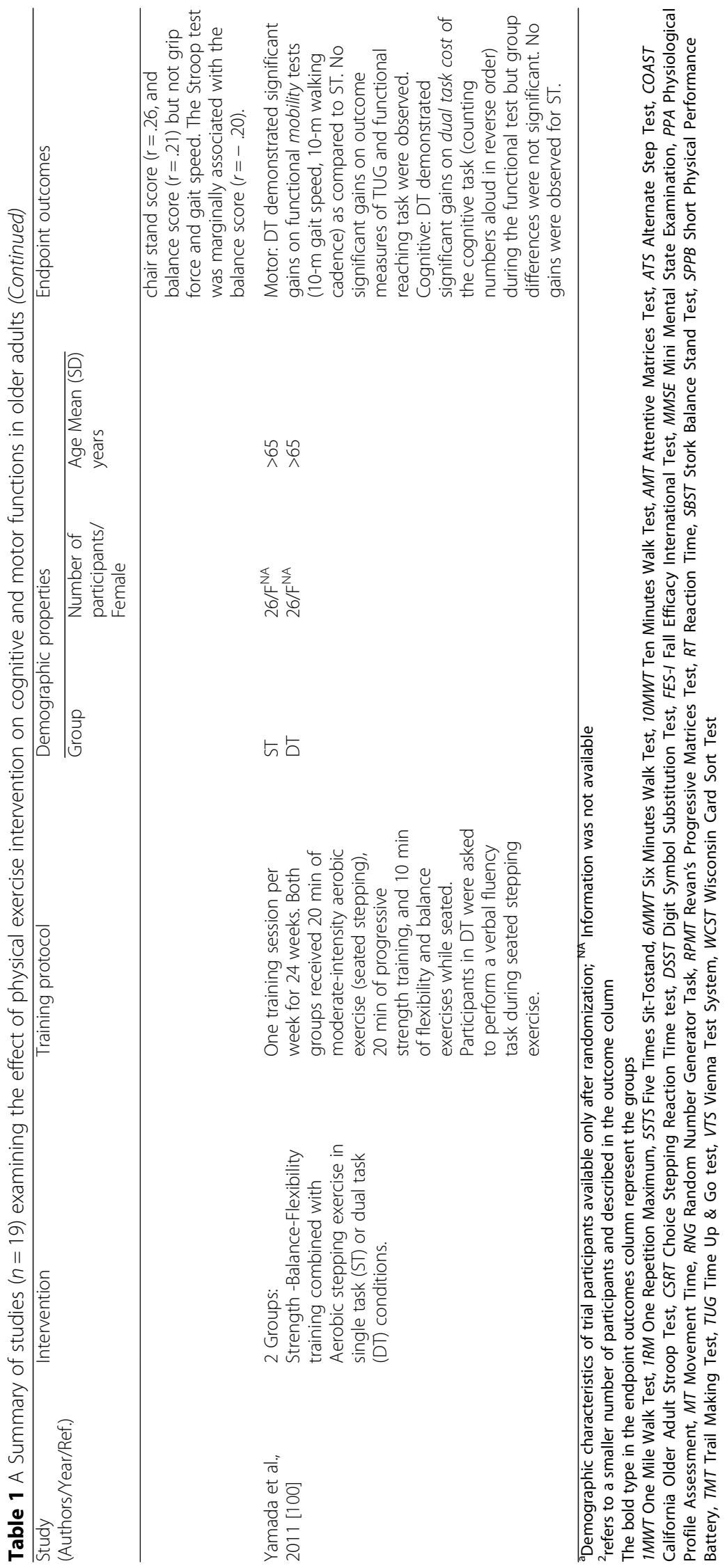


Table 2 Study quality assessment score (Jadad scale [101] with modification ${ }^{\mathrm{a}}$ )

\begin{tabular}{|c|c|c|c|c|}
\hline Study & $\begin{array}{l}\text { Randomization } \\
(\max =2)\end{array}$ & $\begin{array}{l}\text { Blinding }^{a} \\
(\max =2)\end{array}$ & $\begin{array}{l}\text { Account of all participants } \\
(\max =1)\end{array}$ & $\begin{array}{l}\text { Total } \\
(\max =5)\end{array}$ \\
\hline Berryman et al., 2014 [82] & 1 & 0 & 1 & 2 \\
\hline de Bruin et al., 2013 [83] & 2 & 1 & 1 & 4 \\
\hline Falbo et al., 2016 [84] & 1 & 0 & 1 & 2 \\
\hline Fragala et al., 2014 [85] & 1 & 0 & 0 & 1 \\
\hline Granacher et al., 2010 [86] & 1 & 0 & 1 & 2 \\
\hline Hackney et al., 2015 [87] & 0 & 1 & 1 & 2 \\
\hline Hamacher et al., 2015 [88] & 2 & 1 & 1 & 4 \\
\hline Iuliano et al., 2015 [89] & 2 & 0 & 0 & 2 \\
\hline Kamegaya et al., 2014 [90] & 1 & 0 & 1 & 2 \\
\hline Leon et al., 2015 [91] & 1 & 0 & 1 & 2 \\
\hline Maki et al., 2012 [92] & 1 & 0 & 1 & 2 \\
\hline Marmeleira et al., 2009 [93] & 1 & 0 & 1 & 2 \\
\hline Schoene et al., 2013 [94] & 2 & 1 & 1 & 4 \\
\hline Smiley-Oyen et al., 2008 [95] & 0 & 1 & 1 & 2 \\
\hline Theill et al., 2013 [96] & 0 & 0 & 1 & 1 \\
\hline van het Reve \& de Bruin, 2014 [97] & 1 & 0 & 1 & 2 \\
\hline Vaughan et al. 2014 [98] & 2 & 1 & 1 & 4 \\
\hline Williamson et al., 2009 [99] & 2 & 1 & 1 & 4 \\
\hline Yamada et al., 2011 [100] & 2 & 1 & 1 & 4 \\
\hline
\end{tabular}

${ }^{a}$ Since participants cannot be blinded to an exercise intervention, a single-blinded study was awarded 1 point despite the fact that the original JADAD scale require double-blinding in order to receive any point

12 months [99]. In most studies the intervention lasted 8 to 12 weeks and consisted of 24 training sessions (or classes) in total. Exercise protocols also varied greatly between studies. For example, the intensity of the aerobic exercise varied from light (e.g. [94]) to moderatehigh (e.g. [89]). Durations of the training sessions (for all types of interventions) were inconsistent as well, ranging from 15 to $20 \mathrm{~min}$ [89] for balance training to 60-70 $\mathrm{min}$ $[87,88]$ for dance. Similar to the differences in exercise intensity and duration, the type and combinations of exercises varied greatly between studies. For example, three studies that included a combined-exercise training consisted of aerobic-strength training [82, 88, 91], and three studies consisted of strength-balance training [83, 97, $100]$, whereas in five studies all three exercise paradigms were used in a single training session [85, 88, 90, 98, 99]. Finally, six studies that combined physical-cognitive intervention protocols consisted of physical exercise training with a dual task $[84,91,93,94,96,100]$, whereas the interventions in the remaining studies were made up of separate blocks of physical exercise and cognitive training $[83,97]$ or involved social interactions [92]. Exercises in the physical-cognitive intervention consisted of aerobic training [92-94, 96, 100] or combined aerobic/strength/ balance training $[83,84,91,97]$.

\section{Main outcome measures}

Due to the large heterogeneity in exercise protocols and testing methods, it was difficult to arrive at a synthesis of the search findings. Therefore, we performed a descriptive analysis where performance gains (or negative effects) were sorted and summed according to four motor outcome measures and five cognitive outcome measures. The four motor outcome measures were: functional lower limb mobility and gait characteristics [82-84, 86-90, 92-94, 96100], static and/or dynamic balance [86, 87, 89, 94, 97-99], muscle strength [82, 85, 89, 90, 92, 99], and psychomotor (RT) tasks [83, 85, 91, 93-95, 97, 98]. The five cognitive outcome measures were: processing speed [85-99], working memory [82, 84, 88, 90, 92, 95, 96, 98, 99], inhibition [82, 84, 89, 93-96, 98, 99], attention [85, 87-99], and dualtask cost [82-84, 86, 88, 92-94, 96, 97, 100]. Other outcome measures were aerobic fitness [82, 95], depression scores [87, 90, 92], quality-of-life and life-satisfaction scores $[87,90,92]$, and markers of brain plasticity (brain-derived neurotrophic factor - BDNF) $[85,98]$. Battery of tests used for the assessments of the aforementioned motor/cognitive outcome measures in each of the included studies are specified in Table 1.

The outcome effects of each intervention on specific cognitive and motor functions are presented in Table 1. 
Data are summarized in Fig. 2 for the overall motor/cognitive gains in each intervention category, and in Figs. 3 and 4 for the specific motor (Fig.3) and cognitive (Fig. 4) gains in each intervention category. As can be seen in Fig. 2, the highest number of reported performance gains and negative findings were reported for the combined exercise training and cognitive-motor training. However, the abovementioned interventions were also the most frequent (Table 1). In line with the first main objective of the current systematic review, the specific effects of the different intervention categories on motor and cognitive gains are described in detail next.

\section{Motor performance gains as a function of intervention}

Motor performance gains (from a number of studies) are illustrated in Fig. 3 for each of the six interventions. The majority of test batteries (or protocols) examined gains in functional tasks (i.e. mobility and strength) [82, 84, $86-90,92,94,97-100]$, gross motor skills (i.e. balance) [84, 86-89, 94, 96-99], or RT [83, 91, 93-95, 97, 98]. None of the included studies examined fine motor skills or motor learning. Four studies used aerobic [89, 95], strength $[85,89]$,or balance $[86,89]$ training as a single intervention. In one study [89], the three interventions and a passive control group were included in a single study design (see Table 1; [89]). Significant gains induced by aerobic training were found only for a mobility pre/ post-test (gait speed, One Mile Walk Test), significant gains induced by resistance training were found for the strength pre/post-tests (One Repetition Maximum test in all trained muscles), and significant positive gains induced by balance training have been shown only for a balance pre/post-test (Stork Balance Stand Test). No gains on all elements of the test battery were observed in the passive control group. For the remaining studies, aerobic training [95], resistance training [85], or balance training [86] were applied as single interventions. Likely beneficial gains in performance of the visuomotor RT task (i.e. shorter responses times at likelihood of $80.2 \%$ ) were reported in [85] and significant gains in gait stability were reported in [86]. Taken together, observations from the four studies suggest that using aerobic $[89,95]$, strength $[85,89]$, or balance $[86,89]$ training as a single intervention may have only limited effects on motor performance gains. However, findings cannot be generalized due to limitations caused by the small number of studies or the diversity in testing protocols - specifically, no inclusion of psychomotor tests [86, 89] and no inclusion of mobility, balance, and strength tests [85, 95].

Two studies focused on dance as a single exercise $[87,88]$. Observations from these studies are summarized in Fig. 3D. Irrespective of differences in the intervention and testing protocols, both studies showed a significant increase in gait speed. One of these studies also reported a significant increase in backward gait speed as well as faster performance time on the Four-Square Step Test [87].

Eleven studies used combined-exercise training protocols: (i) aerobic and strength $[82,88,91]$, (ii) aerobic and flexibility [90], (iii) strength and balance [83, 95, 97], or (iv) aerobic, strength, and balance exercises combined [84, 88, 98-100]. Observations from these studies are summarized in Fig. 3E. In all studies but two [91, 95], mobility tests were applied pre- and post-intervention, and in seven of nine studies significant gains were observed in one or more mobility performance tests: Time Up \& Go (TUG) [82, 98], Walking Speed [82, 97-100], Stride Length Variability [88], and Chair Stand [82, 97, 99]. Improvements in mobility characteristics were observed for

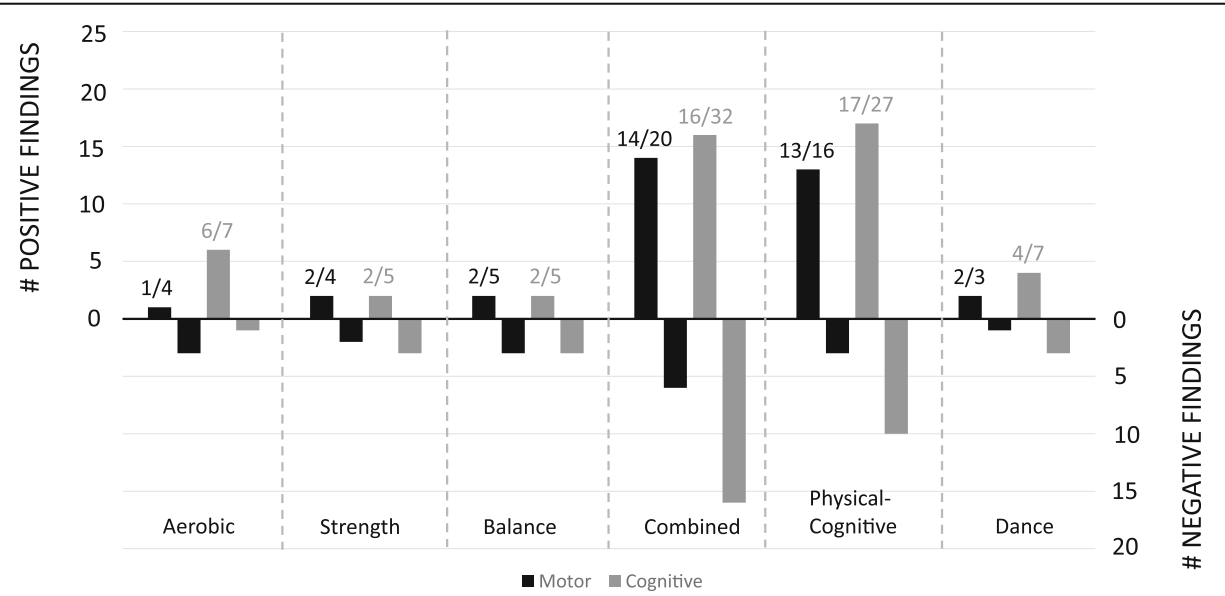

Fig. 2 Outcome effects of each of the six types of interventions on overall motor and cognitive functions. Positive findings refer to significant pre-to-post improvements of performance in one or more of the four main motor outcome measures (i.e. mobility, strength, balance, and psychomotor speed) and one or more of the five main cognitive outcome measures (i.e., attention, processing speed, memory, inhibition, and dual-task cost). Negative findings indicate the number of incidences where no significant gains on the abovementioned outcome measures were found. For specific performance gains see Fig. 3 (motor) and Fig. 4 (cognitive) 

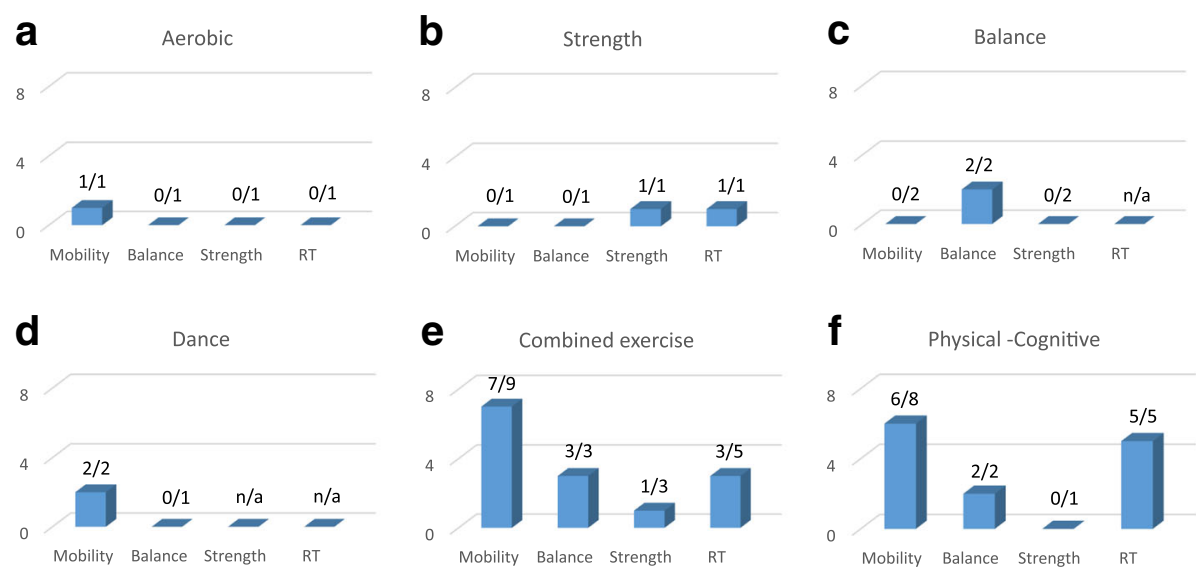

Fig. 3 Outcome effects of each of the six types of interventions on motor performance gains (from the number of studies). Please note that one study can measure more than one outcome. $\mathbf{a}$ aerobic, $\mathbf{b}$ strength, $\mathbf{c}$ balance, $\mathbf{d}$ dance, $\mathbf{e}$ combined exercise, $\mathbf{f}$ physical-cognitive. $\mathrm{n} / \mathrm{a}=$ information was not available

all training protocols in which strength exercises were included [82, 88, 97-100], albeit pre-to-post gains in strength were reported only by one study [82] in which two intervention groups and one control group were tested. Three studies reported significant pre-to-post improvements in balance [97-99] and three of four studies reported significant pre-to-post improvements in the performance of one or more psychomotor tests [83, 91, 97]. However, all three studies that reported pre-to-post improvements in balance also included balance training in their intervention. Finally, pre-to-post gains on RT were found in five studies $[83,91,95,97,98]$. In two of the studies the training protocol consisted of combined strength and balance exercises [83, 97]. The three remaining studies consisted of aerobic-strength training [91] or aerobic-strength-balance training [98].
Nine intervention studies used one or more paradigms of combined physical-cognitive training. Observations from these studies are summarized in Fig. 3F. In six of the nine studies [84, 91, 93, 94, 96, 100], physical and cognitive training were conducted in a dual-task manner. In the remaining studies [83, 92, 97], participants received the cognitive intervention $[83,97]$ or social intervention [92] at the end of the physical training. Intervention protocols consisted of: (i) aerobic exercise combined with: a battery of cognitive-psychomotor training [93], memory training [96], a video game [94]; (ii) strength-balance exercise [83, 97] combined with computerized cognitive training for attention; and (iii) aerobicstrength-balance exercise combined with dual-task interference and/or a battery of psychomotor and memory tasks $[84,91,100]$. Most of the pre-to-post performance gains

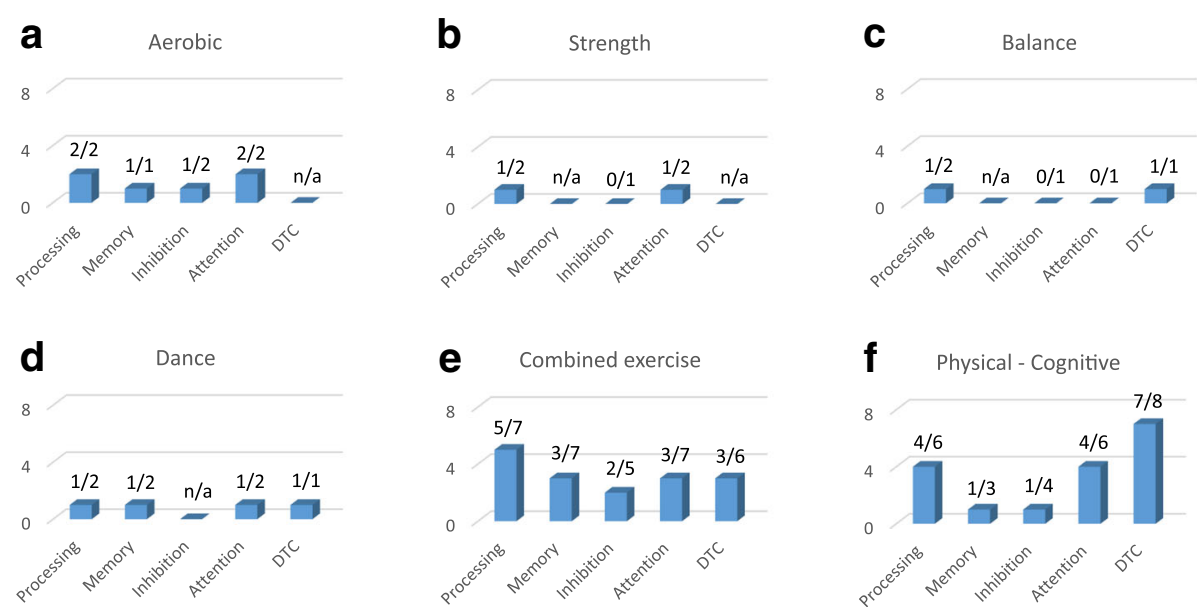

Fig. 4 Outcome effects of each of the six types of interventions on cognitive performance gains (from the number of studies). Please note that one study can measure more than one outcome. $\mathbf{a}$ aerobic, $\mathbf{b}$ strength, $\mathbf{c}$ balance, $\mathbf{d}$ dance, $\mathbf{e}$ combined exercise, $\mathbf{f}$ physical-cognitive. $n / a=$ information was not available 
were reported for mobility outcome measures, specifically TUG [92], walking speed [97, 100], stride length/gait speed variability [84, 96], and chair stand [97]. Significant pre-topost gains were also observed for balance [94, 97]. However, observed gains in the above-mentioned studies were not specific to the intervention, nor to the type of physical exercises or the cognitive training protocols involved. Finally, pre-to-post gains in RT were examined in five studies, in which attention training and/or dual task training exercises were applied [83, 91, 93, 94, 97]. In all five studies a significant improvement in simple RT and/or movement time was observed post-intervention, but significant group differences were evident only when a passive control group was included $[91,93]$. Thus, the existence of an evident link between these two types of cognitive training and respective pre-to-post gains in Stepping Reaction Time (SRT) cannot be generalized. To conclude, intervention protocols using single-exercise training tended to result in focal performance gains $[86,89]$, whereas multiple exercise training [82, 83, 97-99] or physicalcognitive training [83, 94, 97] typically resulted in gains of multiple motor outcome measures.

\section{Cognitive performance gains as function of intervention}

Cognitive performance gains (from the number of studies) are illustrated in Fig. 4 for each of the six interventions. Again, pre-to-post performance gains on cognitive outcome measures were more visible in groups that underwent combined physical exercise training $[82-84,88,90,91,97-100]$ or combined physicalcognitive training $[82,84,91-94,96,97,100]$ than in groups that underwent aerobic training $[89,95]$, strength training $[85,89]$ or balance training $[86,89]$ as a single exercise. Pre-to-post improvements on processing and attention were found in both studies in which aerobic training was applied as a single intervention $[89,95]$, whereas significant improvements on memory [89] or inhibition [95] were evident only in one of the two studies. Finally, the beneficial effects of strength training $[89,95]$ or balance training $[86,95]$ on cognition were marginal, with evidence pointing to possible gains in processing speed [85], attention [89] or motor interference task [86], but not on inhibition $[85,89]$ or cognitive interference task [86] (see, Figs. 4A-C).

The effects of dance as a single intervention on cognitive functions in older adults were reported in two studies $[87,88]$. In one study [87], no pre-to-post gains in cognition were reported. In contrast, the other study [88], which used a cognitive-motor interference task (a serial three subtractions test while walking), found a significant decrease in the average time required to recite the successive subtractions and a marginal increase in the percentage of correct answers, suggesting pre-to-post improvements in processing speed, working memory, attention, and dual-task cost (see, Fig. 4D). Of note, the durations of the single training sessions in both studies were largely similar (90 min including warm-up and cool-down). However, the duration of the intervention was twice as long in one study [88] (26 weeks) than in the other study [87] (12 weeks). This could partially explain the absence of significant post-intervention effects in the latter study.

Findings from the eleven studies in which combinedexercise training protocols were used (see, Fig. 4E) and the nine studies in which combined physical-cognitive training protocols were used (see, Fig. 4F) are discussed next. Due to the large variety among the applied cognitive test batteries, pre-to-post intervention effects are presented for each of the five outcome measures separately, as a function of the different training protocols. Statistically significant pre-to-post-intervention differences on one or more outcome measures of processing were reported for aerobic-flexibility training [90], for strength-balance training [97], and for aerobic-strengthbalance [98]. Improvements were found on: (i) Digit Symbol Substitution Test (DSST) and Analogy test scores [90], (ii) TMT parts A and B time scores [97], and (iii) California Older Adult Stroop Test (COAST) and TMT (parts A and B) time scores [98]. Significant pre-to-post-intervention differences on one or more outcome measures of processing were also reported in four studies in which combined physical-cognitive training protocols were used [91-93, 97]. In three of those studies [92, 93, 97], significant pre-to-post improvements on one or more outcome measures of attention or dualtask cost were also found. Significant pre-to-post differences were found: (i) in TMT parts A and B time scores following strength-balance training and computerized attention training [97]; (ii) on the Categorical Word Fluency element of the 5-Cog test, the digit symbol substitution test (DSST), and the Yamaguchi Kanji symbol substitution tests following aerobic training combined with social interaction [92]; (iii) in a visual processing (Useful Field of View Test (UFOV)) [93], and (iv) in the Simple/Choice RT elements of the Vienna Test System [91].

Statistically significant pre-to-post-intervention differences on one or more outcome measures of memory were reported for aerobic-strength training [82] and aerobic-flexibility training [90]. In [82], improvements in two elements of the Random Generator Number test (RNG, R scores, and mean repetition gap (MRG)) were observed only during a dual task (i.e., performing the RNG test during walking), and were more prominent for the intervention group that underwent aerobic training combined with strength exercise of the upper body muscles (UBS-A group) than in the intervention group that underwent strength exercise of the lower body muscles (LBS-A group). Pre-to-post gains on MRG scores of the RNG test were found only for the UBS-A and control 
groups, but not for the LBS-A. For [90], a significant gain was reported on the Cued Recall Test of the FiveCog task. However, a comparable improvement was also found in the control group. Pre-to-post-intervention improvements in memory were observed in only one [92] of the three studies [84, 92, 96] where memory tests were conducted after the implementation of combined physical-cognitive training.

Statistically significant pre-to-post-intervention differences on one or more outcome measures of inhibition were reported for aerobic-strength training [82] and aerobic-strength-balance training [98]. In one study [82], significant gains were found for the Turning Point Index (TPI, changes between ascending and descending phases) and the adjacency score (numbers presented in pairs; i.e. 3-4) of the RNG test that were visible in both the single-and dual-task test conditions. However, improvements were not statistically different as a function of the group, and were not consistent across test conditions. With respect to the studies in which combined physical-cognitive training protocols were used, pre-topost-intervention improvements in the performance of the inhibition component in the RNG test was reported only in one study following an intervention with dualtask walking [84].

Statistically significant pre-to-post-intervention differences on one or more outcome measures of attention were reported for aerobic-flexibility training [90], strengthbalance training [97], and aerobic-strength-balance training [98]. Testing protocols were not identical across the three abovementioned studies. For [97], significant pre-to-post improvements were reported in the performance of the divided attention task of the Vienna Test System. For [90], a significant gain was reported on the Character Position Referencing task of the Five-Cog task, but a comparable improvement was also found for participants in the passive control group. Finally, [98] reported significant pre-to-post improvement on performance of the TMT parts A and B tests (see also improvement in processing), but not on the Letter-Number Sequencing task. No significant gains were reported by [95] for strength-balance training and for $[88,99]$ for aerobic-strength-balance training.

With respect to the studies in which combined physical-cognitive training protocols were used, significant pre-to-post-intervention improvements on one or more outcome measures of attention were reported in four studies [91-93, 97]. With respect to one study [97], significant pre-to-post improvements were also reported on all divided attention elements of the Vienna Test System. Finally, another study [93] reported significant preto-post improvement in the divided attention element of the UFOV evaluation tool.

Significant pre-to-post-intervention differences on Dual-Task Cost (DTC) were reported by [82] for aerobic- strength training and [83] for strength-balance training (however, statistical power in the latter study was poor due to the small sample size). In [82], improvements in DTC were associated with improvement in working memory and inhibition, as reported above. Interestingly, for three of the six studies mentioned above [91, 97, 100], significant pre-to-post improvements on DTC were reported when the same physical intervention protocols were repeated whilst cognitive training was added. With respect to the studies in which combined physicalcognitive training protocols were used, pre-to-postintervention improvements in DTC were reported in seven of the eight studies where this outcome measure was tested [83, 92-94, 96, 97, 100]. Gains were not specific to the intervention program (either to the type of physical exercises or to the cognitive training protocols involved) or to the evaluation protocol.

\section{Association between motor and cognitive gains}

In line with the second major aim of the current reviewlooking into the dual effect of various training protocols on motor and cognition, we provided a qualitative overview of the extent by which pre-to-post gains in motor functions parallel improvements in the performance of cognitive functions. Specifically, a detailed inspection of the data in Table 1 indicates that parallel improvements in motor and cognitive performances were observed, mainly for interventions consisting of combined physical training or combined physical-cognitive training. The occurrences of parallel improvements in motor and cognitive outcome measures are illustrated in Fig. 5 for the two combined training interventions. It can be seen that parallel improvements were mainly found for: (i) mobility and dual-task cost (DTC) [82, 83, 92, 96, 97, 99, 100], (ii) mobility, balance, processing speed, and attention [97, 98], or (iii) psychomotor speed, processing speed, attention, and/or DCT [83, 91, 93, 94, 97]. To a lesser extent, we also found associations between: (i) mobility (TUG/gait speed/gait variability), balance and inhibition for physical-cognitive training [84, 98], or (ii) between gait speed, strength, and inhibition for combined exercise training [82].

For the remaining interventions (aerobic, strength, balance, and/or dance), parallel improvements in physical/ motor outcome measures and cognitive outcome measures were found between: (i) mobility (gait speed) and processing speed, attention, memory, and DTC in dance [88]; (ii) mobility, attention, and processing speed in aerobic training [89]; (iii) psychomotor speed, attention, and processing speed for strength training [85]; and (iv) balance, processing speed, and DTC in balance training [86]. Overall, these qualitative analyses suggest that prepost gains in gait, mobility, and balance were associated with cognitive improvements. However, most of the 

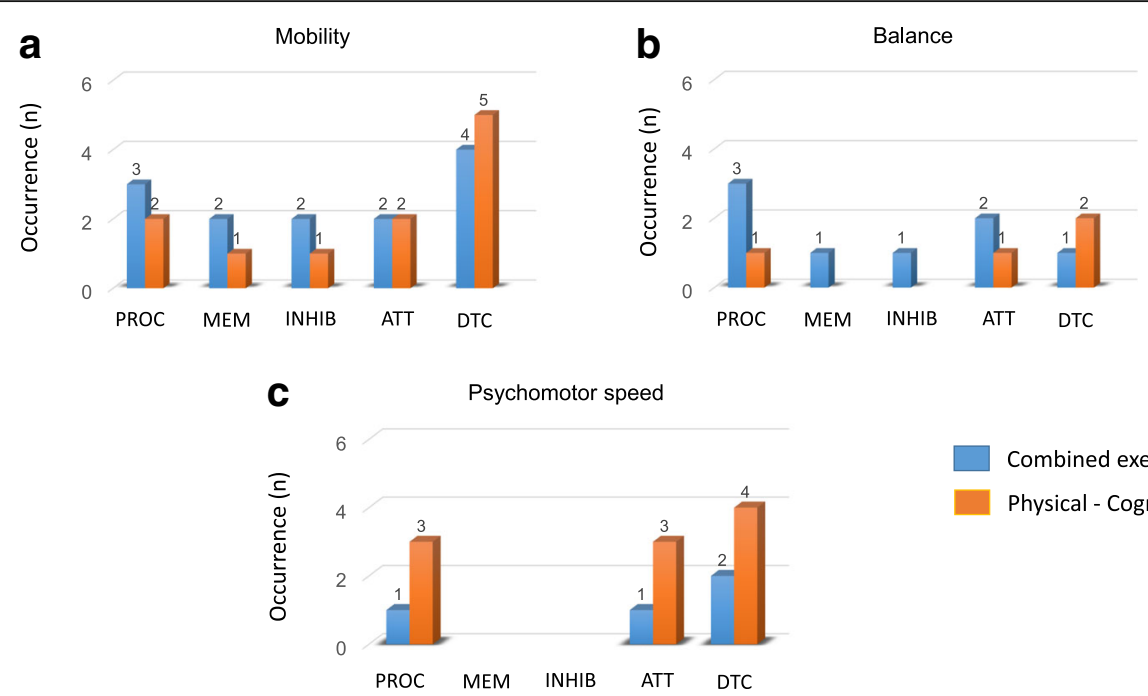

Fig. 5 Occurrences of parallel improvements in motor and cognitive outcome measures for combined exercise training (data extracted from $n=$ 11 studies) and combined physical-cognitive training (data extracted from $n=9$ studies). PROC $=$ processing speed; $M E M=$ memory; $I N H I B=i n h i b i t i o n ;$ ATN = attention; DTC = dual-task cost. Parallel improvements in strength and inhibition were reported only by [82] - data are not shown. See text for the remaining single exercise interventions. a mobility, $\mathbf{b}$ balance, $\mathbf{c}$ psychomotor speed

included studies did not examine correlations between the pre-post difference values of cognitive and motor outcome measures.

Direct assessment of the associations between pre-topost difference values of cognitive and motor outcome measures were available in only two of the nineteen reviewed studies [84, 99]. One study [84] reported that increased inhibition efficiency was associated with decreased gait variability $(r=-.65, p=.006)$ in the group that underwent physical-cognitive dual-task training. However, this effect was found only during dual-task walking with simple gait demands. The same authors reported a marginally significant association between the same outcome measures also for the group that underwent the physical training alone (aerobic-strength-balance combined). Here, a significant correlation between increase inhibition efficiency and decreased gait variability $(r=-.47, p=.049)$ was reported for dual-task walking with complex gait demands (i.e., walking while negotiating hurdles). The second study [99] reported that preto-post gains in processing and attention (as measured with DSST) following aerobic-strength-balance training were positively correlated with improvements in the Short Physical Performance Battery (SPPB) scores $(r=.38, p$ $=.002)$, chair stand scores $(r=.26, p=.012)$, and to some extent balance scores $(r=.21, p=047)$. The same authors also reported significant positive correlations between gains on short-term memory scores (Rey Auditory Verbal Learning Test, (RAVLT) ) and gait velocity $(r=0.25, p$ $=.019)$ or chair stand $(r=.22, p=.039)$. Otherwise, correlations between pre-to-post changes in the performance of RAVLT or the inhibition test (Stroop), and pre-to-post changes in all other elements of the SPPB, did not reach the level of significance $(r \leq .20, p>.05)$.

\section{Discussion}

In line with the objectives of the current systematic review, the search strategy we used aimed at locating research studies that examined the combined effects of physical training interventions on motor and cognitive functions in older adults. Our literature search and selection process resulted in 19 publications, of which 11 studies reported the effects of combined (multi-component) exercise training [82-84, 88, 90, 91, 97-100], 9 reported the effects of combined physical cognitive training [83, 84, 91-94, 96, 97, 100], and 8 reported the effects of single exercise protocols with aerobic training $[89,95]$, strength training $[85,89]$, balance training $[86,89]$, or dance $[87,88]$. The main findings from the 19 included studies were:

1. Multi-component exercise training or combined physical-cognitive training were found to improve a larger number of physical, motor, and cognitive outcome measures than a single exercise intervention. Physical-cognitive training was found to be the best intervention strategy.

2. Multi-component exercise training was found to be beneficial for improving gait and processing speed, whereas combined physical-cognitive training was found to be most beneficial for psychomotor speed, processing speed, attention, and dual task cost.

3. Pre-post gains in mobility and psychomotor speed were strongly associated with pre-to-post gains in 
processing and dual task cost. However, these associations were more prevalent when intervention consisted of combine physical-cognitive training.

4. Due to lack of sufficient consistency in the training protocols and applied test batteries, we were unable to provide a reliable evaluation of the possible effects of single-exercise protocols on performance.

\section{Specificity of the exercise interventions}

The extent to which exercise interventions were associated with specific gains in cognitive and motor functions were examined in line with the first major aim of the current systematic review. Overall, findings from the nineteen included studies indicated that combined exercise training and physical-cognitive training resulted in significant improvements in mobility (e.g. increased walking speed and reduced time of chair rise), attention, and processing capabilities. Also, improvements in psychomotor speed and dual-tasks cost were more pronounced after physical-cognitive training than after exercise training alone. The aforementioned observations are in line with findings from previous systematic reviews or meta-analysis studies [45, 46], all together providing evidence that multi-component exercise training or combined physical-cognitive training appeared to be the best intervention strategies for improving multiple physical, motor, and cognitive functions. This augmented effect could be attributed to parallel improvements in processing and attention, which were more evident after combined physical-cognitive training than after combined exercise training. Moreover, observations from the nineteen included studies indicated that combined physical-cognitive training had a greater beneficial effect than other types of interventions on processing and attention, but not on inhibition and memory (e.g. [91-93, 97]); partly explaining the observed improvements in the performances of dualtask when this type of intervention was used. However, the above-mentioned findings need to be interpreted with caution, due to the large diversity among the intervention protocols and testing methods.

Pre-to-post improvements in mobility, processing, attention, and/or dual-task cost were also evident in the control group, which underwent physical exercise training or cognitive training as a single intervention (e.g. $[95,96])$. The fact that non-significant time $\times$ group interactions were observed, indicates that training effects on some of the outcome measures may not necessarily be specific to the training protocol, but may have been caused by merely engaging in physical activity once or twice a week. For example, in five of the nine studies that used combined physical-cognitive training [83, 84, 91, 97, 100], pre-to-post gains in mobility outcome measures were statistically similar to the respective gains found in the control groups that underwent physical training alone.
Moreover, the performance gains observed in participants who received the training were not always statistically different from the gains observed in participants in the control groups who attended health education classes [92, 99] or received cognitive training alone [96]. Nonetheless, significant group differences were observed in seven of the nine studies where a passive control group was included [85, 86, 89, 91, 93, 94, 96]. Taken together, one could suggest that adding cognitive elements to the physical intervention may have only a minor additional effect on the mobility characteristics at the post-tests. However, a closer inspection of the findings indicated that improvements in mobility were associated to a greater extent with pre-post gains in dual-task cost after combined physical-cognitive training than after exercise training alone (e.g. [84]). From a brain-behavior perspective, parallel improvements in mobility characteristics and dual-task cost may suggest improvements in the functioning of the basal ganglia and prefrontal cortex $[2,5,50,102]$.

Significant gains in mobility or functional motor tests (e.g., chair rise) could be attributed, at least in part, to significant gains in cardiovascular performance (e.g., [59]; for studies included in the present review see $[82,95])$ or increase of muscle strength $[29,31]$. The findings from studies included in the current review [84, 98-100] suggest that multimodal combined training would likely lead to greater benefits for general health, cardio-respiratory fitness, and general improvement of cognitive and motor functions than aerobic, strength, or balance training alone. Nonetheless, due to the diversity in interventions and test protocols among the nineteen included studies, we were unable to make a clear association between the types of training used and their specific effects on performance.

\section{Cognitive-motor interactions}

Intervention effects on both cognitive and motor functions were examined, in line with the second major aim of the current systematic review. Findings from the nineteen included studies suggest that intervention effects on mobility, balance, and psychomotor speed were associated with improvements in attention, processing, and dual-tasks (Fig. 5). Intervention effects on mobility and balance, together with improvements in inhibition or memory, were also observed, but were less evident. Importantly, parallel improvements in physical (motor) and cognitive outcome measures were observed in the majority of the intervention groups $(75 \%)$ that underwent combined physical-cognitive training, but only in about $35 \%$ of the groups that underwent physical-exercise training only. This observation suggests, at first sight, that positive training effects (in both motor and cognitive function) might be attributed exclusively to the inclusion of cognitive training; specifically dual-task 
training $[84,91,93,94,96,100]$; see for further evidence $[27,29,31]$. Taken together, the aforementioned findings suggest that the beneficial effects of physical-cognitive training (in general) and dual task training (in particular) appeared to be superior to other forms of training protocols. However, a closer inspection of the findings indicated that training effects on dual-task performance occurred in parallel to pre-to-post gains in attention, processing, and psychomotor speed (e.g. [97]). Moreover, parallel improvement in physical or cognitive functions under dual-tasks were also evident (albeit to a lesser extent) in studies that used physical exercise training as the main intervention (e.g. [82]), and improvements in physical performance tasks were found when performed under single-task conditions (e.g. [86]). To conclude, the main body of evidence from the current systematic review suggests that combined exercise and cognitive training (in particular when the cognitive training consists of a dual task) could improve basic cognitive and motor functions, and give rise to better management of brain resources $[25,59,102-104]$. This observation is not surprising given the fact that training under a dual task requires sustained attention to visual or auditory stimuli, effective processing of sensory information, and effective transfer of information among the brain's sensory centers. As such, we expect that this type of intervention would infiltrate high-order executive-control centers and sensorimotor centers, causing neuroplastic changes in widespread areas of the aging brain, as compared to other types of interventions which may induce more local effects. These findings must be interpreted with caution, however, given the low number of included studies and large variety in the intervention and test protocols.

The association between changes in inhibition and mobility or inhibition and balance could be attributed partly to the beneficial effects of cardiovascular training [95]; see for further evidence [103, 105]. However, evidence from other included studies that also applied cardiovascular training either exclusively or solely [89], or in combination with other physical/cognitive exercises [93, 96], failed to support this assumption. Notably, positive training effects on attention could indicate adaptation of a shared attention-inhibition substructure, for example the dorsolateral prefrontal cortex or the prefrontal-basal ganglia network $[6,102,103]$. This could entail selective benefits for attention and inhibition or inhibition and gait performance under a dual task. However, associations between changes in inhibition and attention [98] or inhibition and dual-task walking [84] were rather scarce, as compared to associations between basic cognitive functions and mobility or psychomotor speed (Fig. 5).

Negative findings were reported in the majority $(69 \%)$ of the included studies where pre-to-post training effects on inhibitory functions were examined [89, 93-96, 99]; see also [84] for combined exercise training but not combined physical-cognitive training. The absence of consistent findings on inhibition across the included studies could be attributed to the large diversity among training protocols, intervention durations, and assessment tools. However, the fact that most of the included studies did report significant training effects when preto-post differences were examined for attention (61\%), processing (67\%), and dual-task costs (75\%), implies that some training protocols showed selective beneficial effects for inhibition, where others did not. In line with these observations, we propose that inhibition (and possibly also memory) may be responsive to specific types of training paradigms, whereas other basic cognitive (or motor) functions such as attention or mobility may be responsive to a broader range of interventions or multimodal training protocols. Similar to the findings from two other systematic reviews $[18,20]$, findings from the current review suggest that multimodal interventions have a greater beneficial effect on older adults than do single interventions - specifically, improving a broader range of cognitive-motor functions and having a better potential protective effect on the structural and functional integrity of the aging brain. Further insights into the effects of specific training protocols on pre-to-post differences in brain-behavior relationships should be considered in future research by including brain imaging techniques.

\section{Brain-behavior relationships}

While evidence from other research studies or systematic reviews could provide some indications about traininginduced reorganization of the brain ([26, 32, 51-55]; see reviews $[20,22])$, none of the included studies in this review included direct measurements of training-induced differences in brain structure. Indirect evidence for possible relationships between cognitive and motor performance gains and brain plasticity have been examined, nonetheless, in two of the nineteen included studies [85, 98], based on the measurement of brain-derived neurotrophic factor (BDNF) levels in serum or plasma; yet, these observations were inconsistent. Specifically, one study [98] reported a significant increase in the levels of plasma BDNF in response to a combined physical exercise intervention which included cardiovascular, strength, and motor fitness training. The same authors reported a decrease in BDNF levels in participants of the passive control group, which did not receive any exercise training during the period of the intervention. This was also the observation from other studies, where an increased BDNF level in older adults has been reported in response to physical exercise [106, 107], dance [52], or combined physical-cognitive training [106]; see review [20]. In 
addition, it was found that individuals who experienced greater fitness improvements from the exercise training (i.e., high responders to exercise) also had greater increases in the serum neurotrophic factors, such as BDNF and insulin-like growth factor-1 (IGF-1) [106].

In contrast to the aforementioned observations, one study [85] reported no significant change of serum BDNF in response to a resistance training intervention. Yet evidence also suggests that increases in serum neurotrophic factors appeared to be less responsive to resistance training as compared to other exercise interventions; see [18] for a systematic review and metaanalysis. Furthermore, inconsistencies in findings could be attributed to differences in gender and intensity/duration of the intervention $[18,51,107]$ or individual difference in baseline levels of BDNF $[18,108]$.

Notably, changes in levels BDNF (or other neurotrophic factors) could provide a general indication for neuroplasticity, but cannot provide indications about the exact brain regions or networks that were affected by the intervention. For that reason, studies comparing two or more modes of interventions against a single mode (or controls) should also include pre/post measurements of brain structures. Including neuroimaging data in future studies will be important in order to examine changes in gray or white matter, or brain metabolic profiles to examine the effect of an intervention on neurotransmitter concentration or integrity of brain tissue) at specific brain regions. Some studies have already taken this step [26, 32, 51, 53]. However, most studies reported cross-sectional associations between self-reported physical activity and gray matter or white matter volume (see, for review [20]). Therefore, in future studies, assessments should be made of: (i) the extent to which the brain structure and functions are influenced by different types of interventions, and (ii) the extent to which brain structural and functional changes occur along with preto-post intervention changes in motor and cognitive measurements assessed before and after exercise.

\section{Limitations}

The present systematic review has several limitations. First, the included studies applied very heterogeneous intervention protocols and test batteries, which limited our ability to gain conclusive insights into the specific training effect of each type of intervention - in particular, the lack of consistency among the outcome measures tested in each study and the use of different test batteries for assessment of the same outcome measure. In addition, we found some mismatches between the physical fitness components of training and the reported outcome measures, especially in studies where combined physical-cognitive training protocols were used. For example, nine of eleven studies included strength exercises in the combined-exercise training protocol, but only three studies performed pre- and post-intervention tests of strength [82, 90, 99]. Taken together, this large diversity in methodology hindered our ability to compare results from different studies and perform a quantitative meta-analysis.

Second, all included studies in the current review reported pre-to-post intervention gains on multiple outcome measures. But in none of the included studies were adjustments for multiple testing across dependent variables made; post-hoc comparisons for significant main effects from analyses of variance or covariance, or mixed model regressions within each dependent variable were adjusted (for the most part) by using the Bonferroni correction [82, 84, 86, 89-92, 95]. However, this does not address the issue of Type-1 error for testing across multiple dependent variables; see for example [82, $87,89,92-94,99,100]$. Therefore, we encourage scientists in this area to adapt a more conservative approach for evaluating their findings; for example, to discuss multivariate results at the $p<.001$ level or greater (e.g. [109]) or to apply a false discovery rate procedure [110].

Other limitations may pertain to the use of a single data-base source (PubMed) and/or the elimination of studies which included patient groups. However, PubMed is considered to be a reliable source, and offers free access to most research articles, meta-analysis papers, and systematic reviews. Therefore, it is most likely that very few studies, if at all, may be found in other scientific sources. The inclusion of patient groups may, on the one hand, allow more specific insights into mechanisms or brain structures which may benefit from the intervention. On the other hand, variability among patients regarding the type and severity of their pathological conditions is expected, complicating the synthesis of the search findings.

\section{Conclusions}

Findings from the nineteen included studies indicated that the majority of training effects affected mobility. The same training protocols also appeared to improve attention, processing, and dual-task cost to a greater degree than inhibition and memory. In line with findings from other studies that examined the effects of multimodal combined training on cognitive functions, observations from the studies included in our systematic review indicate that simultaneous training of cognitive and physical abilities has the highest potential to induce simultaneous gains in motor cognitive abilities. Unfortunately, none of the included studies in this review examined a parallel effect of training on brain plasticity, albeit findings from one study [98] reported a significant increase in the levels of plasma BDNF as a result of the intervention. More research is required to determine the 
exact effects of cognitive-physical training on structural and functional changes in specific brain areas, as well as on interactions between functionally interconnected brain networks. Finally, we encourage scientists in this area to develop specific and consistent test batteries for assessing cognitive and motor effects of exercise. This will enable a clearer picture of the effects of exercise, and will make it possible to conduct reviews and draw general conclusions.

\section{Abbreviations}

10MWT: Ten Minutes Walk Test; 1MWT: One Mile Walk Test; 1RM: One Repetition Maximum; 5STS: Five Times Sit-Tostand; 6MWT: Six Minutes Walk Test; AMT: Attentive Matrices Test; ATS: Alternate Step Test; COAST: California Older Adult Stroop Test; CSRT: Choice Stepping Reaction Time test; DNF: Brain-derived neurotrophic factor; DSST: Digit Symbol Substitution Test; DTC: Dual-Task Cost; FES-I: Fall Efficacy International Test; LBS-A: Lower Body Strength \& Aerobics; MMSE: Mini Mental State Examination; MRG: Mean Repetition Gap; MT: Movement Time; PPA: Physiological Profile Assessment; RAVLT: Rey Auditory Verbal Learning Test; RNG: Random Number Generator Task; RPMT: Revan's Progressive Matrices Test; RT: Reaction Time; SBST: Stork Balance Stand Test; SPPB: Short Physical Performance Battery; SRT: Stepping Reaction Time; TMT: Trail Making Test; TUG: Time Up \& Go Test; UBSA: Upper Body Strength \& Aerobics; UFOV: Useful Field of View Test; VTS: Vienna Test System; WCST: Wisconsin Card Sort Test

\section{Acknowledgements}

The authors would like to thank Dinah Olswang for her help during the preparation of this manuscript.

\section{Funding}

No funding was used during the preparation of this review.

\section{Availability of data and materials}

Not applicable.

\begin{abstract}
Authors' contributions
OL: concept creation, bibliography systematic search, establishing the exclusion/ inclusion criteria, design of the review, categories formation, creating the Table and Figures, writing. YN: collaborating in concept creation, assistance in the design of the article, and in categories formation, assistance in decisions making on inclusion/exclusion of articles, proof-reading the manuscript. GZ: collaborating in bibliography systematic search, collaborating in screening articles for inclusion/ exclusion, assistance in writing and in designing the Table, Critically reading and proof-reading the manuscript.
\end{abstract}

Ethics approval and consent to participate

Not applicable.

\section{Consent for publication}

Not applicable.

\section{Competing interests}

None of the authors have any competing interests that are related to this review.

\section{Publisher's Note}

Springer Nature remains neutral with regard to jurisdictional claims in published maps and institutional affiliations.

\section{Author details}

'Movement Control and Neuroplasticity Research Group, Biomedical Sciences Group, Department of Movement Sciences, KU Leuven, Heverlee, Belgium. ${ }^{2}$ The Academic College at Wingate, Netanya, Israel.
Received: 22 September 2017 Accepted: 5 December 2017

Published online: 21 December 2017

\section{References}

1. Boisgontier MP, Cheval B, van Ruitenbeek P, Levin O, Renaud O, Chanal J, et al. Whole-brain grey matter density predicts balance stability irrespective of age and protects older adults from falling. Gait Posture. 2016;45:143-50.

2. Boisgontier MP, Cheval B, Chalavi S, van Ruitenbeek P, Leunissen I, Levin O, et al. Individual differences in brainstem and basal ganglia structure predict postural control and balance loss in young and older adults. Neurobiol Aging. 2017:50:47-59.

3. Coxon JP, Goble DJ, Van Impe A, De Vos J, Wenderoth N, Swinnen SP. Reduced basal ganglia function when elderly switch between coordinated movement patterns. Cereb Cortex. 2010;20(10):2368-79.

4. Coxon JP, Van Impe A, Wenderoth N, Swinnen SP. Aging and inhibitory control of action: Cortico-subthalamic connection strength predicts stopping performance. J Neurosci. 2012;32(24):8401-12.

5. Demnitz N, Zsoldos E, Mahmood A, Mackay CE, Kivimäki M, Singh-Manoux A, et al. Associations between mobility, cognition, and brain structure in healthy older adults. Front Aging Neurosci. 2017;9

6. Eckert MA, Keren NI, Roberts DR, Calhoun VD, Harris KC. Age-related changes in processing speed: unique contributions of cerebellar and prefrontal cortex. Front Hum Neurosci. 2010;4

7. Fleischman DA, Yang J, Arfanakis K, Arvanitakis Z, Leurgans SE, Turner AD, et al. Physical activity, motor function, and white matter hyperintensity burden in healthy older adults. Neurology. 2015:84(13):1294-300.

8. Fujiyama H, Van Soom J, Rens G, Gooijers J, Leunissen I, Levin O, et al. Agerelated changes in frontal network structural and functional connectivity in relation to bimanual movement control. J Neurosci. 2016;36(6):1808-22.

9. Kennedy KM, Raz N. Aging white matter and cognition: differential effects of regional variations in diffusion properties on memory, executive functions, and speed. Neuropsychologia. 2009;47(3):916-27.

10. Raz N, Gunning-Dixon FM, Head D, Dupuis JH, Acker JD. Neuroanatomical correlates of cognitive aging: evidence from structural magnetic resonance imaging. Neuropsychology. 1998;12(1):95-114.

11. Ruscheweyh R, Deppe M, Lohmann H, Wersching H, Korsukewitz C, Duning $T$, et al. Executive performance is related to regional gray matter volume in healthy older individuals. Hum Brain Mapp. 2013;34(12):3333-46.

12. Van Petten C. Relationship between hippocampal volume and memory ability in healthy individuals across the lifespan: review and meta-analysis. Neuropsychologia. 2004;42(10):1394-413.

13. Van Petten C, Plante E, Davidson PSR, Kuo TY, Bajuscak L, Glisky EL. Memory and executive function in older adults: relationships with temporal and prefrontal gray matter volumes and white matter hyperintensities. Neuropsychologia. 2004;42(10):1313-35.

14. Eckert MA. Slowing down: age-related neurobiological predictors of processing speed. Front Neurosci. 2011;5

15. Glisky EL. Changes in cognitive function in human aging. In: Riddle DR, editor. Brain aging: models, methods, and mechanisms. Boca Raton, FL: CRC Press; 2007. p. 3-20.

16. Levin O, Fujiyama H, Boisgontier MP, Swinnen SP, Summers JJ. Aging and motor inhibition: a converging perspective provided by brain stimulation and imaging approaches. Neurosci Biobehav Revs. 2014;43:100-17.

17. Seidler RD, Bernard JA, Burutolu TB, Fling BW, Gordon MT, Gwin JT, et al. Motor control and aging: links to age-related brain structural, functional, and biochemical effects. Neurosci Biobehav Rev. 2010;34(5):721-33.

18. Barha CK, Davis JC, Falck RS, Nagamatsu LS, Liu-Ambrose T. Sex differences in exercise efficacy to improve cognition: a systematic review and metaanalysis of randomized controlled trials in older humans. Front Neuroendocrinol. 2017:46:71-85.

19. Buckner RL. Memory and executive function in aging and AD. Neuron. 2004 44(1):195-208

20. Erickson Kl, Leckie RL, Weinstein AM. Physical activity, fitness, and gray matter volume. Neurobiol Aging. 2014;35(suppl 2):S20-8.

21. Kelly ME, Loughrey D, Lawlor BA, Robertson IH, Walsh C, Brennan S. The impact of exercise on the cognitive functioning of healthy older adults: a systematic review and meta-analysis. Ageing Res Rev. 2014;16:12-31.

22. Voelcker-Rehage C, Niemann C. Structural and functional brain changes related to different types of physical activity across the life span. Neurosc. Biobehav Rev. 2013;37(9):2268-95. 
23. Martins RA, Coelho E, Silva MJ, Pindus DM, Cumming SP, Teixeira AM, Veríssimo MT. Effects of strength and aerobic-based training on functional fitness, mood and the relationship between fatness and mood in older adults. J Sports Med Phys Fitness. 2011;51(3):489-96.

24. González-Palau F, Franco M, Bamidis PD, Losada R, Parra E, Papageorgiou SG, et al. The effects of a computer-based cognitive and physical training program in a healthy and mildly cognitive impaired aging sample. Aging Ment Health. 2014;18(7):838-46.

25. Chapman SB, Aslan S, Spence JS, Defina LF, Keebler MW, Didehbani N, et al. Shorter term aerobic exercise improves brain, cognition, and cardiovascular fitness in aging. Front Aging Neurosci. 2013;5

26. Chapman SB, Aslan S, Spence J, Keebler MW, DeFina LF, Didehbani N, et al. Distinct brain and behavioral benefits from cognitive versus physical training: a randomized trial in aging adults. Front Hum Neurosci. 2016;10(338):1-15.

27. Eggenberger $P$, Wolf $M$, Schumann $M$, de Bruin ED. Exergame and balance training modulate prefrontal brain activity during walking and enhance executive function in older adults. Front Aging Neurosci. 2016;8

28. Boucard GK, Albinet CT, Bugaiska A, Bouquet CA, Clarys D, Audiffren M. Impact of physical activity on executive functions in aging: a selective effect on inhibition among old adults. J Sport Exerc Psychol. 2012;34(6):808-27.

29. Desjardins-Crépeau L, Berryman N, Fraser SA, TTM V, Kergoat MJ, Li KZH, et al. Effects of combined physical and cognitive training on fitness and neuropsychological outcomes in healthy older adults. Clin Interv Aging. 2016;11:1287-99.

30. Duchesne C, Lungu $O$, Nadeau A, Robillard ME, Boré A, Bobeuf F, et al Enhancing both motor and cognitive functioning in Parkinson's disease: aerobic exercise as a rehabilitative intervention. Brain Cogn. 2015;99:68-77.

31. Forte R, Boreham CAG, Leite JC, De Vito G, Brennan L, Gibney ER, et al. Enhancing cognitive functioning in the elderly: multicomponent vs resistance training. Clin Interv Aging. 2013;8:19-27.

32. Niemann C, Godde B, Staudinger UM, Voelcker-Rehage C. Exercise-induced changes in basal ganglia volume and cognition in older adults. Neuroscience. 2014;281:147-63.

33. Predovan D, Fraser SA, Renaud M, Bherer $L$. The effect of three months of aerobic training on stroop performance in older adults. J Aging Res. 2012;2012

34. Rosano C, Venkatraman VK, Guralnik J, Newman AB, Glynn NW, Launer L, et al. Psychomotor speed and functional brain MRI 2 years after completing a physical activity treatment. J Gerontol - Ser a biol Sci. Med Sci. 2010;65(6):639-47.

35. Colcombe $S$, Kramer AF. Fitness effects on the cognitive function of older adults: a meta-analytic study. Psychol Sci. 2003;14(2):125-30.

36. Beauchet O, Launay C, Annweiler C, Fantino B, Allali G, De Decker L. Physical training-related changes in gait variability while single and dual tasking in older adults: magnitude of gait variability at baseline matters. Eur J Phys Rehabil Med. 2013;49(6):857-64

37. Eggenberger $P$, Theill N, Holenstein S, Schumacher $V$, de Bruin ED. Multicomponent physical exercise with simultaneous cognitive training to enhance dual-task walking of older adults: a secondary analysis of a 6-month randomized controlled trial with I-year follow-up. Clin Interv Aging. 2015;10:1711-32.

38. Hartmann A, Murer $K$, de Bie RA, de Bruin ED. The effect of a training program combined with augmented afferent feedback from the feet using shoe insoles on gait performance and muscle power in older adults: a randomised controlled trial. Disabil Rehabil. 2010;32(9):755-64.

39. Mian OS, Baltzopoulos V, Minetti AE, Narici MV. The impact of physical training on locomotor function in older people. Sport Med. 2007;37(8):683-702.

40. Mian OS, Thom JM, Ardigò LP, Morse Cl, Narici MV, Minetti AE. Effect of a 12-month physical conditioning programme on the metabolic cost of walking in healthy older adults. Eur J Appl Physiol. 2007;100(5):499-505.

41. Bakken RC, Carey JR, Di Fabio RP, Erlandson TJ, Hake JL, Intihar TW. Effect of aerobic exercise on tracking performance in elderly people: a pilot study. Phys Ther. 2001;81(12):1870-9.

42. Kattenstroth JC, Kalisch T, Holt S, Tegenthoff M, Dinse HR. Six months of dance intervention enhances postural, sensorimotor, and cognitive performance in elderly without affecting cardio-respiratory functions. Front Aging Neurosci. 2013;5

43. Keogh JW, Morrison S, Barrett R. Strength training improves the tri-digit finger-pinch force control of older adults. Arch Phys Med Rehabil. 2007; 88(8):1055-63.

44. Olafsdottir HB, Kim SW, Zatsiorsky VM, Latash ML. Anticipatory synergy adjustments in preparation to self-triggered perturbations in elderly individuals. J Appl Biomech. 2008;24(2):175-9.
45. Cadore EL, Rodríguez-Mañas $L$, Sinclair A, Izquierdo M. Effects of different exercise interventions on risk of falls, gait ability, and balance in physically frail older adults: a systematic review. Rejuvenation Res. 2013;16(2):105-14.

46. Hortobágyi T, Lesinski M, Gäbler M, VanSwearingen JM, Malatesta D, Granacher U. Effects of three types of exercise interventions on healthy old adults' gait speed: a systematic review and meta-analysis. Sports Med. 2015; 45(12):1627-43

47. Bauer E, Toepper M, Gebhardt H, Gallhofer B, Sammer G. The significance of caudate volume for age-related associative memory decline. Brain Res. 2015; 1622:137-48.

48. Aron AR, Durston S, Eagle DM, Logan GD, Stinear CM, Stuphorn V. Converging evidence for a fronto-basal-ganglia network for inhibitory control of action and cognition. J Neurosci. 2007;27(44):11860-4.

49. DeLong M, Wichmann T. Update on models of basal ganglia function and dysfunction. Park Relat Disord. 2009;15(Suppl 3):s237-40.

50. Boisgontier MP, van Ruitenbeek P, Leunissen I, Chalavi S, Sunaert S, Levin O, et al. Nucleus accumbens and caudate atrophy predicts longer action selection times in young and old adults. Hum Brain Mapp. 2016;37(12):4629-39.

51. Liu-Ambrose T, Nagamatsu LS, Voss MW, Khan KM, Handy TC. Resistance training and functional plasticity of the aging brain: a 12-month randomized controlled trial. Neurobiol Aging. 2012;33(8):1690-8.

52. Müller P, Rehfeld K, Schmicker M, Hökelmann A, Dordevic M, Lessmann V, et al. Evolution of neuroplasticity in response to physical activity in old age: the case for dancing. Front Aging Neurosci. 2017;9

53. Rehfeld K, Müller P, Aye N, Schmicker M, Dordevic M, Kaufmann J, et al. Dancing or fitness sport? The effects of two training programs on hippocampal plasticity and balance abilities in healthy seniors. Front Hum Neurosci. 2017;11

54. Tseng BY, Gundapuneedi T, Khan MA, Diaz-Arrastia R, Levine BD, Lu H, et al. White matter integrity in physically fit older adults. Neurolmage. 2013;82:510-6.

55. Weinstein AM, Voss MW, Prakash RS, Chaddock L, Szabo A, White SM, et al. The association between aerobic fitness and executive function is mediated by prefrontal cortex volume. Brain Behav Immun. 2012;26(5):811-9.

56. Pfefferbaum A, Adalsteinsson E, Sullivan EV. Frontal circuitry degradation marks healthy adult aging: evidence from diffusion tensor imaging. Neurolmage. 2005;26(3):891-9.

57. Bettcher BM, Mungas D, Patel N, Elofson J, Dutt S, Wynn M, et al. Neuroanatomical substrates of executive functions: beyond prefrontal structures. Neuropsychologia. 2016;85:100-9.

58. Bossers WJR, van der Woude LHV, Boersma F, Hortobágyi T, Scherder EJA, van Heuvelen MJG. A 9-week aerobic and strength training program improves cognitive and motor function in patients with dementia: a randomized, controlled trial. Am J Geriatr Psychiatry. 2015;23(11):1106-16.

59. Voelcker-Rehage C, Godde B, Staudinger UM. Cardiovascular and coordination training differentially improve cognitive performance and neural processing in older adults. Front Hum Neurosci. 2011;5

60. Plummer P, Zukowski LA, Giuliani C, Hall AM, Zurakowski D. Effects of physical exercise interventions on gait-related dual-task interference in older adults: a systematic review and meta-analysis. Gerontology. 2015;62(1):94-117.

61. Masley S, Roetzheim R, Gualtieri T. Aerobic exercise enhances cognitive flexibility. J Clin Psychol Med Settings. 2009;16(2):186-93.

62. Agmon M, Kelly VE, Logsdon RG, Nguyen H, Belza B. The effects of EnhanceFitness (EF) training on dual-task walking in older adults. J Appl Gerontol. 2015;34(3):NP128-42.

63. Donath L, Faude O, Bridenbaugh SA, Roth R, Soltermann M, Kressig RW, et al. Transfer effects of fall training on balance performance and spatiotemporal gait parameters in healthy community-dwelling older adults: a pilot study. J Aging Phys Act. 2014;22(3):324-33.

64. Dorfman M, Herman T, Brozgol M, Shema S, Weiss A, Hausdorff J, et al. Dual-task training on a treadmill to improve gait and cognitive function in elderly idiopathic fallers. J Neurol Phys Ther. 2014;38(4):246-53.

65. Cruz-Ferreira A, Marmeleira J, Formigo A, Gomes D, Fernandes J. Creative dance improves physical fitness and life satisfaction in older women. Res Aging. 2015;37(8):837-55.

66. Ito S, Hashimoto M, Aduma S, Yasumura S. Effectiveness of locomotion training in a home visit preventive care project: one-group pre-intervention versus post-intervention design study. J Orthop Sci. 2015;20(6):1078-84.

67. Lu X, Siu KC, Fu SN, Hui-Chan CWY, Tsang WWN. Tai chi practitioners have better postural control and selective attention in stepping down with and without a concurrent auditory response task. Eur J Appl Physiol. 2013;113(8): 1939-45. 
68. Marini M, Monaci M, Manetti M, Piazza M, Paternostro F, Sgambati E. Can practice of Dancesport as physical activity be associated with the concept of "successful aging"? J Sports Med Phys Fitness. 2015;55(10):1219-26.

69. Wollesen B, Voelcker-Rehage C, Willer J, Zech A, Mattes K. Feasibility study of dual-task-managing training to improve gait performance of older adults. Aging Clin Exp Res. 2015;27(4):447-55.

70. WL W, Wei T. Sen, Chen SK, Chang JJ, Guo LY, Lin HT. the effect of Chinese yuanji-dance on dynamic balance and the associated attentional demands in elderly adults. J sport. Sci Med. 2010;9(1):119-26.

71. Best JR, Chiu BK, Liang Hsu C, Nagamatsu LS, Liu-Ambrose T. Long-term effects of resistance exercise training on cognition and brain volume in older women: results from a randomized controlled trial. J Int Neuropsychol Soc. 2015;21(10):745-56

72. Chang Y-K, Huang C-J, Chen K-F, Hung T-M. Physical activity and working memory in healthy older adults: an ERP study. Psychophysiology. 2013; 50(11):1174-82.

73. Dechamps A, Diolez $\mathrm{P}$, Thiaudière $\mathrm{E}$, Tulon A, Onifade $\mathrm{C}$, Vuong $\mathrm{T}$, et al. Effects of exercise programs to prevent decline in health-related quality of life in highly deconditioned institutionalized elderly persons: a randomized controlled trial. Arch Intern Med. 2010;170(2):162-9.

74. Hamacher D, Hamacher D, Rehfeld K, Schega L. Motor-cognitive dual-task training improves local dynamic stability of normal walking in older individuals. Clin Biomech. 2016;32:138-41.

75. Gregory MA, Gill DP, Zou G, Liu-Ambrose T, Shigematsu R, Fitzgerald C, et al. Group-based exercise combined with dual-task training improves gait but not vascular health in active older adults without dementia. Arch Gerontol Geriatr. 2016;63:18-27.

76. Irvine AB, Gelatt VA, Seeley JR, Macfarlane P, Gau JM. Web-based intervention to promote physical activity by sedentary older adults: randomized controlled trial. J Med Internet Res. 2013;15(2)

77. Kimura K, Obuchi S, Arai T, Nagasawa H, Shiba Y, Watanabe S, et al. The influence of short-term strength training on health-related quality of life and executive cognitive function. J Physiol Anthropol. 2010;29(3):95-101.

78. Ota A, Yasuda N, Horikawa S, Fujimura T, Ohara H. Differential effects of power rehabilitation on physical performance and Higherlevel functional capacity among community-dwelling older adults with a slight degree of frailty. J Epidemiol. 2007;17(6):587-92.

79. Pichierri G, Coppe A, Lorenzetti S, Murer K, de Bruin ED. The effect of a cognitive-motor intervention on voluntary step execution under single and dual task conditions in older adults: a randomized controlled pilot study. Clin Interv Aging. 2012;7:175-84.

80. Pichierri G, Murer K, de Bruin EDA. Cognitive-motor intervention using a dance video game to enhance foot placement accuracy and gait under dual task conditions in older adults: a randomized controlled trial. BMC Geriatr. 2012;12(1):74

81. Tsang WWN, Hui-Chan CWY, Effects FSN. Of tai chi on pre-landing muscle response latency during stepping down while performing a concurrent mental task in older adults. Eur J Appl Physiol. 2012;112(7):2663-9.

82. Berryman N, Bherer L, Nadeau S, Lauzière S, Lehr L, Bobeuf F, et al. Multiple roads lead to Rome: combined high-intensity aerobic and strength training vs. gross motor activities leads to equivalent improvement in executive functions in a cohort of healthy older adults. Age. 2014;36(5):9710-9.

83. de Bruin ED, van het Reve E, Murer KA. Randomized controlled pilot study assessing the feasibility of combined motor-cognitive training and its effect on gait characteristics in the elderly. Clin Rehabil. 2013;27(3):215-25.

84. Falbo S, Condello G, Capranica L, Forte R, Pesce C. Effects of physicalcognitive dual task training on executive function and gait performance in older adults: a randomized controlled trial. Biomed Res Int. 2016;2016

85. Fragala MS, Beyer KS, Jajtner AR, Townsend JR, Pruna GJ, Boone CH, et al. Resistance exercise may improve spatial awareness and visual reaction in older adults. J strength Cond Res. 2014;28(8):2079-87.

86. Granacher U, Muehlbauer T, Bridenbaugh S, Bleiker E, Wehrle A, Kressig RW. Balance training and multi-task performance in seniors. Int J Sports Med. 2010;31(5):353-8.

87. Hackney ME, Byers C, Butler G, Sweeney M, Rossbach L, Bozzorg A. Adapted tango improves mobility, motor-cognitive function, and gait but not cognition in older adults in independent living. J Am Geriatr Soc. 2015; 63(10):2105-13.

88. Hamacher D, Hamacher D, Rehfeld K. H??Kelmann a, Schega L. the effect of a six-month dancing program on motor-cognitive dual-task performance in older adults. J Aging Phys Act. 2015;23(4):647-52.
89. Iuliano E, di Cagno A, Aquino G, Fiorilli G, Mignogna P, Calcagno G, et al. Effects of different types of physical activity on the cognitive functions and attention in older people: a randomized controlled study. Exp Gerontol. 2015;70:105-10.

90. Kamegaya T, Araki Y, Kigure H, Yamaguchi H. Twelve-week physical and leisure activity programme improved cognitive function in community-dwelling elderly subjects: a randomized controlled trial. Psychogeriatrics. 2014;14(1):47-54.

91. León J, Ureña A, Bolaños MJ, Bilbao A, Oña AA. Combination of physical and cognitive exercise improves reaction time in persons 61-84 years old. J Aging Phys Act. 2015;23(1):72-7.

92. Maki Y, Ura C, Yamaguchi T, Murai T, Isahai M, Kaiho A, et al. Effects of intervention using a community-based walking program for prevention of mental decline: a randomized controlled trial. J Am Geriatr Soc. 2012;60(3):505-10.

93. Marmeleira JF, Godinho MB, Fernandes OM. The effects of an exercise program on several abilities associated with driving performance in older adults. Accid Anal Prev. 2009:41(1):90-7.

94. Schoene D, Lord SR, Delbaere K, Severino C, Davies TA, Smith STA. Randomized controlled pilot study of home-based step training in older people using videogame technology. PLoS One. 2013;8(3):e57734.

95. Smiley-Oyen AL, Lowry KA, Francois SJ, Kohut ML, Ekkekakis P. Exercise, fitness, and neurocognitive function in older adults: the "selective improvement" and "cardiovascular fitness" hypotheses. Ann Behav Med. 2008:36(3):280-91.

96. Theill N, Schumacher V, Adelsberger R, Martin M, Jäncke L. Effects of simultaneously performed cognitive and physical training in older adults. BMC Neurosci. 2013;14:103.

97. van het Reve E, de Bruin ED. Strength-balance supplemented with computerized cognitive training to improve dual task gait and divided attention in older adults: a multicenter randomized-controlled trial. BMC Geriatr. 2014;14:134

98. Vaughan S, Wallis M, Polit D, Steele M, Shum D, Morris N. The effects of multimodal exercise on cognitive and physical functioning and brainderived neurotrophic factor in older women: a randomised controlled trial. Age Ageing. 2014;43(5):623-9.

99. Williamson JD, Espeland M, Kritchevsky SB, Newman AB, King AC, Pahor M, et al. Changes in cognitive function in a randomized trial of physical activity: results of the lifestyle interventions and independence for elders pilot study. J Gerontol - Ser a biol Sci. Med Sci. 2009;64(6):688-94.

100. Yamada M, Aoyama T, Tanaka B, Nagai K, Ichihashi N. Seated stepping exercise in a dual-task condition improves ambulatory function with a secondary task: a randomized controlled trial. Aging Clin Exp Res. 2011;23(5):386-92.

101. Jadad AR, Moore RA, Carroll D, Jenkinson C, Reynolds DJM, Gavaghan DJ, et al. Assessing the quality of reports of randomized clinical trials: is blinding necessary? Control Clin Trials. 1996;17(1):1-12.

102. Wong CN, Chaddock-Heyman L, Voss MW, Burzynska AZ, Basak C, Erickson $\mathrm{Kl}$, et al. Brain activation during dual-task processing is associated with cardiorespiratory fitness and performance in older adults. Front Aging Neurosci. 2015;

103. McGregor KM, Nocera JR, Sudhyadhom A, Patten C, Manini TM, Kleim JA, et al. Effects of aerobic fitness on aging-related changes of interhemispheric inhibition and motor performance. Front Aging Neurosci. 2013;5

104. Godde B, Voelcker-Rehage C. Cognitive resources necessary for motor control in older adults are reduced by walking and coordination training. Front Hum Neurosci. 2017;11:156.

105. McGregor KM, Zlatar Z, Kleim E, Sudhyadhom A, Bauer A, Phan S, et al. Physical activity and neural correlates of aging: a combined TMS/fMRI study. Behav Brain Res. 2011;222(1):158-68.

106. Heisz JJ, Clark IB, Bonin K, Paolucci EM, Michalski B, Becker S, et al. The effects of physical exercise and cognitive training on memory and neurotrophic factors. J Cogn Neurosci. 2017; Epub ahead of print.

107. Ruscheweyh R, Willemer C, Krüger K, Duning T, Warnecke T, Sommer J, et al Physical activity and memory functions: an interventional study. Neurobiol Aging. 2011;32(7):1304-19.

108. Barha CK, Galea LA, Nagamatsu LS, Erickson Kl, Liu-Ambrose T. Personalising exercise recommendations for brain health: considerations and future directions. Br J Sports Med. 2017;51(8):636-9

109. French SA, Story M, Neumark-Sztainer D, Fulkerson JA, Hannan P. Fast food restaurant use among adolescents: associations with nutrient intake, food choices and behavioral and psychosocial variables. Int J Obes. 2001;25(12): 1823-33.

110. Curran-Everett D. Multiple comparisons: philosophies and illustrations. Am J Physiol - Regul Integr comp. Physiol. 2000;279(1):R1-8. 